\title{
ANÁLISE DE SISTEMAS DE MESOESCALA UTILIZANDO DADOS DE DESCARGAS NUVEM-TERRA
}

\author{
Sanjar Abdoulaev ${ }^{1}$, Valdo S. Marques ${ }^{1}$, Francisca M. A. Pinheiro ${ }^{1}$, Eduardo \\ F. A. Martinez ${ }^{1} \&$ Olga Lenskaia ${ }^{2}$
}

Received November 05, 2000 / Accepted December 16, 2001

\begin{abstract}
Campos Acumulados de Descargas atmosféricas à terra (CAD) são examinados como fonte na diagnose da estrutura e evolução dos sistemas de mesoescala observados por satélite e radar associados com 16 passagens de frentes e 9 casos de ciclogênese local, durante o inverno de 1999, na região sul do Brasil e do Oceano Atlântico. As maiores taxas de descargas nuvem - terra são associadas à ciclogênese local, em média, 4 (quatro) vezes maior do que as taxas de raio associadas às frentes frias. Construindo a sobreposição dos CAD numa imagem de vídeo, em intervalos de tempo de 0,5 à 2 horas, e código de cores apropriado, foi determinado o seguinte: a) orientação e velocidade das frentes frias, representadas por faixas lineares estreitas ou arcos não acentuados; b) seções frontais (quente e fria) do ciclone e onda frontal, reconhecidas pela sua forma convexa e côncava; c) convecção em desenvolvimento e dissipação com as mesmas características nas imagens de satélite em infravermelho, discriminadas pela forma de CAD e polaridade preferencial das descargas. A análise de 37 sistemas de mesoescala classificados em 6 (seis) padrões de eco de radar, indica que a taxa de raios oscila periodicamente durante a evolução do sistema, crescendo com a extensão da altura de eco de $Z>30 \mathrm{dBZ}$ em direção às temperaturas negativas. $A$ área de eco $>30 \mathrm{dBZ}$ dos sistemas, com núcleos de refletividade maior do que $50 \mathrm{dBZ}$ e alturas de $30 \mathrm{dBZ}$ maiores do que $7,5 \mathrm{~km}$, produz mais do que 0,025 descarga. $\mathrm{h}^{-1} \cdot \mathrm{km}^{-2}$. Os sistemas lineares e não lineares das tempestades severas e os lineares moderados acompanhados por regiões estratiformes extensas produzem número de descargas maior do que em outros sistemas.
\end{abstract}

Palavras-chave: Descarga atmosféricas à terra; Radar; Satélite; Sistemas de mesoescala nas frentes; Ciclones

ANALYSIS OF MESOSCALE SYSTEM USING CLOUD-TO-GROUND FLASH
DATA - Time-Integrated Cloud-to-Ground Lightning Field (TILF) are used as a
source to diagnose the structure and evolution of mesoscale rainfall systems
observed by radar and satellite during cold front movement (16 cases) and local
cyclogenesis (9 cases) southward of Brazilian and South Atlantic Ocean. The
major cloud-to-ground lightning events are associated with local cyclogenesis,
when hourly registered number of flashes is four times greater than frontal cases.
Building the TILF with time intervals of 0.5 to 2 hours and superposed one by
one, using appropriated color-coded display, are determinate as following: a)
direction and velocity of cold fronts, showing narrow bands or not accentuates
arcs; b) warm and cold sections of fronts associated with cyclones and cyclonic
waves, showing their convex and concave shape; c) developing and dissipating
convection having same characteristics on satellite image, but different form of
TILF and preferentialflash polarity. The analysis of 37 mesoscale systems classified
using 6 morphologic types of radar echoes, indicated that lightning rate have
periodic oscillation during system evolution, increasing with extent of height of
30 dBZ towards negative temperatures. The area of systems with echoes $>30$ 
$d B Z$, reflectivity cores more than $50 \mathrm{dBZ}$ and $30 \mathrm{dBZ}$ at height greater than $7.5 \mathrm{~km}$, have flash density more than 0.025 flashes.$h^{-1} \cdot \mathrm{km}^{-2}$. The lightning rates of linear and nonlinear severe storm or moderate bands accompanied by large stratiform regions are stronger than others.

Key words: Cloud-to-ground flashes; Radar; Satellite; Mesoscale systems on fronts; Cyclones.

\author{
${ }^{1}$ Laboratório de Engenharia e Exploração de Petróleo, Universidade Estadual do Norte Fluminense, \\ Sistema de Meteorologia do Estado do Rio de Janeiro, \\ SIMERJ, Rua Real Grandeza, 219, Bloco O, sala 208, \\ 22-283-900, Rio de Janeiro, RJ;

\section{INTRODUÇÃO}

A previsão da atividade elétrica de uma tempestade local ainda é um problema não resolvido. Em primeiro lugar, ainda não são conhecidos com clareza nem a dinâmica de uma tempestade unicelular de cerca de $10 \mathrm{~km}$ de tamanho e vida de meia à 1 hora, nem o processo em que essas células aglomeram-se em sistemas maiores formando os chamados sistemas de mesoescala de precipitação, de centenas $\mathrm{km}$ de extensão e tempo de vida de algumas horas à um dia. Por outro lado, não é completa a teoria física da separação das cargas negativas e positivas em gotas e cristais num pequeno volume no interior da nuvem (ex. em $\mathrm{Cb}$ ou Ns). A descarga elétrica de nuvem à terra, ocorrendo em conseqüência de processos que variam desde a escala macro até a microescala, é um fenômeno de vários estágios (ex. Mason, 1970), um dos quais é uma avalanche de elétrons, a partir da nuvem em direção à terra, no canal do raio pré-ionizado pelo primeiro líder escalonado, produzindo a descarga principal. Este fenômeno também é denominado descarga de retorno, pois a frente de plasma aquecida pela avalanche eletrônica propaga-se rapidamente da terra à nuvem, dando uma noção visual de que a descarga ocorre em direção à nuvem. $\mathrm{O}$ pico de corrente na descarga de retorno, que pode trazer à terra carga negativa ou positiva, alcança valores $>100$ kA (20-30 kA são correntes mais freqüentes), e é acompanhado por forte emissão das ondas eletromagnéticas nas faixas visível (i.é relâmpago) e de radio (i.é. atmosféricos).

A sistematização (Matveev, 1984; Mazin \& Khargian, 1989, entre outros) de observações de atmosféricos, que se iniciaram nos anos 1930s, e de relâmpagos nas estações meteorológicas permitiram estabelecer os seguintes elementos da variação sazonal e regional das descargas à terra e dos raios no interior das nuvens:

1) a taxa de raios cresce com a extensão vertical das nuvens e com a diminuição da temperatura. Há indicativos de que os sistemas de mesoescala organizados (i.é. complexos convectivos, supercélulas, vide Cotton \& Anthes, 1989) produzem maior número de raios do que as tempestades locais.

2) As tempestades com raios concentram-se nos continentes e ilhas, numa faixa entre $20^{\circ} \mathrm{Ne} 20^{\circ} \mathrm{S}$, sendo que a duração das trovoadas é maior no hemisfério sul. Embora a duração de relâmpagos, durante o ano, cresce em direção ao equador, alcançando 800 horas em "capitais dos raios" (e. g. bacia do Rio Amazonas, África equatorial, ilha de Madagascar, arquipélago Sondes), a quantidade relativa de descargas à terra em relação às descargas entre nuvens diminui numa relação de cerca de 1:8, em contraste com a relação de $1: 1$, nas latitudes em torno de $60^{\circ}$. No Brasil, de acordo com Gin \& Guedes (2000), as cidades equatoriais de Manaus, São Luís e Belém, que têm o maior índice anual de relâmpagos em relação a outras localidades apresentam, entretanto, uma variação significativa em número de relâmpagos observados entre o verão e o inverno. A região de maior número de relâmpagos na faixa tropical da América do Sul localizada entre $15^{\circ} \mathrm{S}$ e $20^{\circ} \mathrm{S}$, oscila sazonalmente entre os estados de Goiás $\left(47^{\circ} \mathrm{W}\right)$ e Mato Grosso $\left(57^{\circ} \mathrm{W}\right)$. Na faixa subequatorial, em torno de $10^{\circ} \mathrm{S}$, são observadas também oscilações 
intrasazonais de relâmpagos seguidos de mudanças dos ventos predominantes (Silva Dias, 2001). Durante a estação chuvosa (Janeiro e Fevereiro), em Rondônia, os períodos dos ventos de oeste em $850 \mathrm{hPA}$, associados a passagens de sistemas de grande escala a sudeste e centrooeste do Brasil, o número de raios é quatro vezes menor do que nos períodos intermediários de vento de leste e precipitação isolada.

3) Nas latitudes continentais de $40^{\circ}$ a $70^{\circ}$ as atividades de raios são elevadas durante o verão, desaparecendo no inverno. A maior parte das tempestades no período de inverno nessa faixa latitudinal está nas regiões de ciclogênese e frontogênese das regiões costeiras e dos oceanos a sotavento dos continentes coincidindo, via de regra, com as zonas de confluência das correntes marítimas quentes e frias (e.g. encontro da corrente fria do Labrador e corrente quente Golfstream, procedente do Golfo do México, o que produz gradientes da temperatura do mar em torno de $10^{\circ} \mathrm{C}$ por $100 \mathrm{~km}$ ).

$\mathrm{O}$ estudo pioneiro da atividade elétrica dos sistemas sinóticos atuados na zona de ciclogênese do Atlântico Norte (Ludlam, 1980; p.386), no período de 1940-1950, usando-se a rede britânica de detecção dos raios, localizou os atmosféricos a 1000 $\mathrm{km}$ da ilhas britânicas, com precisão de $100 \mathrm{~km}$. Entre outros fatos importantes foi observado que os raios tendem a se concentrar, em maior número, próximo ao topo da onda ciclonal, do lado da linha da frente quente em superfície. No ciclone maduro, parcialmente ocluso, o maior número de atmosféricos ocorre próximo ao ponto de oclusão e atrás da frente fria. Foi notada, também, a variação latitudinal dos atmosféricos ao longo da frente fria orientada longitudinalmente. $\mathrm{O}$ máximo de atividade elétrica observou-se em torno das latitudes intermediarias de $45^{\circ} \mathrm{N}$, onde foram detectados $30 \%$ das descargas, contrastando com $1 \%$ nas latitudes altas $\sim 60^{\circ} \mathrm{N}$, ou $\operatorname{com} 3 \%$ nas latitudes baixas $\sim 35^{\circ} \mathrm{N}$.

Análogo à zona ciclogenética descrita acima, que influi fortemente nas condições de tempo no Sul do Brasil durante o inverno austral (junho-setembro), há também uma zona de formação de ciclones, localizada nas latitudes subtropicais do Atlântico Sul, onde ocorre a confluência entre as corrente das Malvinas, fria, oriunda do sul do continente, com a corrente do Brasil, quente. Durante o inverno e o outono, na plataforma continental próxima ao litoral do Uruguai e do Rio Grande do Sul, entre $30^{\circ} \mathrm{S}$ e $35^{\circ} \mathrm{S}$, as águas superficiais formam uma frente oceânica com os gradientes térmicos maiores do que $4^{\circ} \mathrm{C}$ por 100 km (e.g. Zavialov et al., 1999). Ainda nessa região, observações recentes com satélite de orbita polar (e.g. Christian e Latham, 1998) mostraram maior taxa relativa de relâmpagos na estação de inverno do que no verão. $O$ monitoramento e a análise da estrutura das frentes e ciclones extratropicais no sudeste da América do Sul, com resolução temporal admissível para previsão de curto prazo, em torno de meia hora, é restrito ao uso de imagens de satélites meteorológicos geoestacionários, cuja resolução espacial é sujeita à degradação gradual com afastamento da área de interesse do ponto sub satélite. No entanto, em muitos esquemas operacionais não se dispõe de uma seqüência temporal de imagens de 30 em 30 minutos (e.g. durante o período dos furacões no hemisfério norte a imagem completa do hemisfério sul é transmitida pelo satélite GOES-8 somente a cada 3 horas).

Além de problemas técnicos inevitáveis, existe a evolução natural dos sistemas de precipitação que se deslocam no extremo sul do Brasil durante o inverno com velocidades de cerca de $20 \mathrm{~m} / \mathrm{s}$ ou mais (Abdoulaev et al., 1998), complicando a identificação do mesmo sistema nas imagens de satélite de baixa resolução temporal. O radar meteorológico fornece informação de resolução excelente (e.g. 15 min, 2'2 $\mathrm{km})$ sobre a estrutura dos sistemas de precipitação e a sua dinâmica. A área onde essa informação pode ser coletada, entretanto, é limitada pelo raio de abrangência útil do radar, de cerca de $300 \mathrm{~km}$. Uma opção alternativa ao satélite e a rede de radares para acompanhamento da evolução dos sistemas no sudeste da América do Sul e Oceano Atlântico Sul, surgiu recentemente com a organização da Rede Integrada de detecção das Descargas Atmosféricas - RIDAT, (Benetti et al., 2000), integrando 22 pontos com sensores de deteç̧ão de raios operados pela Companhia Energética de Minas Gerais (CEMIG, Fig. 1a), Furnas Centrais Elétricas (FURNAS) e pelo Sistema Meteorológico do Paraná (SIMEPAR). Em 
18 locais, o sistema de detecção baseia-se na determinação de instantes de aparecimento e da amplitude do pulso eletromagnético irradiado pela descarga de retorno (em inglês Time Of Arrival $T O A$ ) que permite o cálculo do ponto de interseção das trajetórias hiperbólicas de pulso sobre a superfície esférica da Terra. Em 4 desses locais, além do sensores TOA, estão instalados os chamados detetores de direção (em inglês Direction Finder-DF), que detectam o azimute do raio, comparando as tensões e polaridades relativas induzidas pelo pulso eletromagnético de raio num par de antenas ortogonais de polarização vertical. A latitude e longitude do raio nesse sistema são determinadas pela triangulação, comparando-se os azimutes obtidos nas três estações. Em contraste com os estudos pioneiros (e.g. Pinto et al., 1996; Gin et al., 1996, 1998) das propriedades físicas das descargas elétricas atmosféricas nas tempestades de verão no Sudeste do Brasil (e.g. variação temporal e espacial da densidade e polaridade das correntes elétricas, multiplicidade nas descargas, etc.), ou estudos climáticos baseados nas observações usando rede relativamente densa de detetores de raios (Gin et. al., 2000), o objetivo deste trabalho é verificar a possibilidade do acompanhamento operacional dos sistemas de mesoescala de precipitação na região sudeste da América do Sul (ao sul do estado de Paraná) e Oceano Attântico Sul. A probabilidade de detecção da descarga de retorno pela rede nessas regiões, situadas de 200 à $400 \mathrm{~km}$ da RIDAT, segundo relatório de FURNAS (1999), é menor do que $20 \%$ e, neste contexto, serão preestabelecidas as condições limiares de uso dos dados de descargas em função da escala e do tipo do sistema a ser acompanhado, bem como das possíveis técnicas de integração dos campos de descargas à terra com os dados de radar e satélite.

\section{DADOS E METODOLOGIA}

Para a análise da atividade elétrica do sistema precipitante no extremo sul do Brasil, numa distancia significativa dos detetores de raios da RIDAT, foram separados 30 dias durante o outono e inverno de 1999, quando houve as observações no radar meteorológico de banda S (DWSR-88S) da
Universidade Federal de Pelotas, $\left(31^{\circ} 43^{\prime} \mathrm{W}\right.$, $52^{\circ} 18^{\prime} \mathrm{W}$, vide Fig. 1a). Nesses dias, o radar foi ativado para observação da evolução dos sistemas precipitantes, quando as imagens de vídeo de radar, alcançando raios de 60 a $480 \mathrm{~km}$, foram filmadas fornecendo o campo horizontal e vertical da refletividade $Z$, com seis níveis de refletividade $(Z<29$, 29, 35(40), 45, 50 e >55(60) dBZ) codificados em cores. Na rede interna de FURNAS (Tabela 1) foram disponibilizados 24 arquivos diários de descargas do período observado. Uma linha de arquivo diário descompactado pelo sistema LTraX (real-time Lightning Tracking software) do Global Atmospheric In., contém o número seqüencial de raios a partir do inicio da gravação, o momento de ocorrência da descarga de retorno (CG) com precisão 0,1 segundo, sua localização até $0,001^{\circ}$ de latitude e longitude, polaridade (positiva $+\mathrm{CG}$ ou negativa $-\mathrm{CG}$ ) e pico da corrente na descarga em $\mathrm{kA}$.

As sondagens atmosféricas realizadas no aeroporto de Porto-Alegre e campos meteorológicos em níveis padrão às 0000 GMT gerada pelo National Center for Enviromental Prediction (NCEP), foram usados para verificar a situação meteorológica no Rio Grande do Sul (RS) observada nas imagens do satélite GOES-8 do National Oceanic and Atmospheric Administration (NOAA), nos canais infravermelho e de vapor d'água. Nessas imagens foram determinadas as frentes transientes, apresentando uma linha de nuvens de mais de $300 \mathrm{~km}$ com deslocamento visível, e situação da ciclogênese local com a nebulosidade ciclônica permanecendo sobre o RS por mais de 12 horas.

Condicionalmente, a amostra estudada foi dividida em:

a) 9 casos do ciclogênese localizados sobre RS (e.g. casos 10, 18, 21-23 na tabela 1);

b) 16 sistemas frontais, sendo que 9 passando rapidamente pelo Rio Grande do Sul, na direção NE (e.g. casos 11, 14), ou para o interior do estado (e.g. casos 6, 9) e 2 sistemas estacionários sobre o noroeste do RS (4) e no sul do Uruguai (19). Os 2 sistemas frontais interagiram com vórtice ciclônico (casos 3,26) e em 2 dias os raios foram associados com a zona de instabilidade pré-frontal. Os casos 11 e 12 foram adicionados à amostra inicial, para completar a 

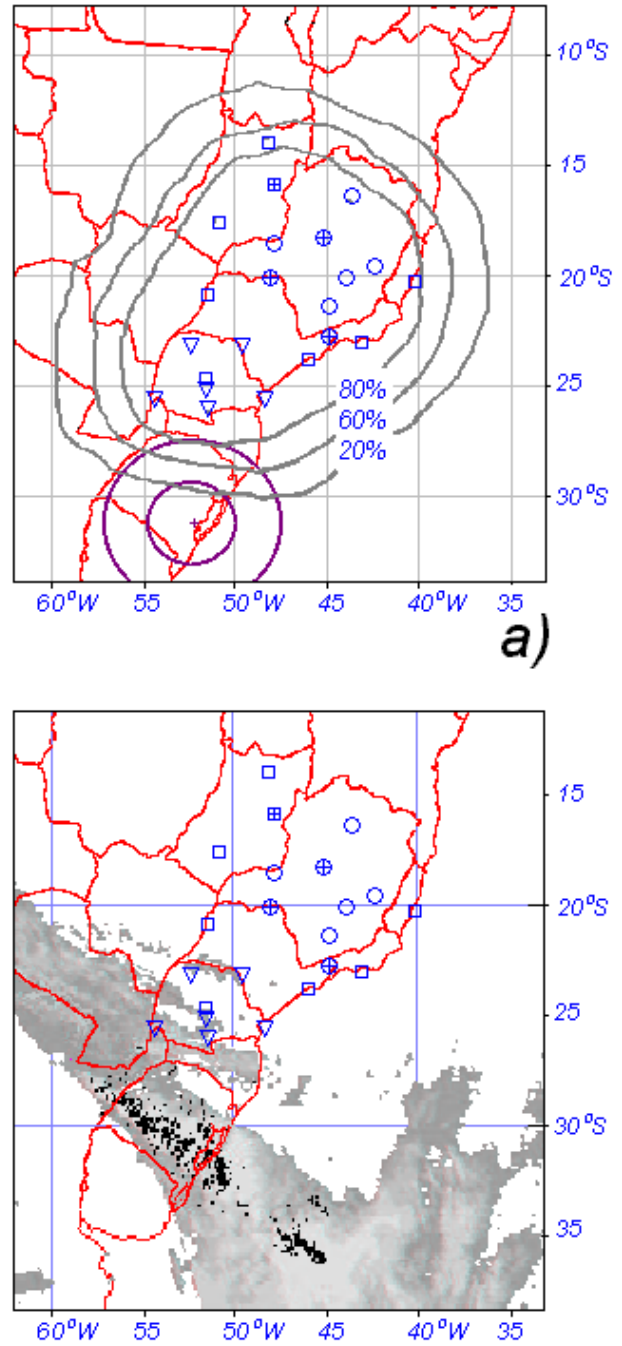

0845-0915

b)

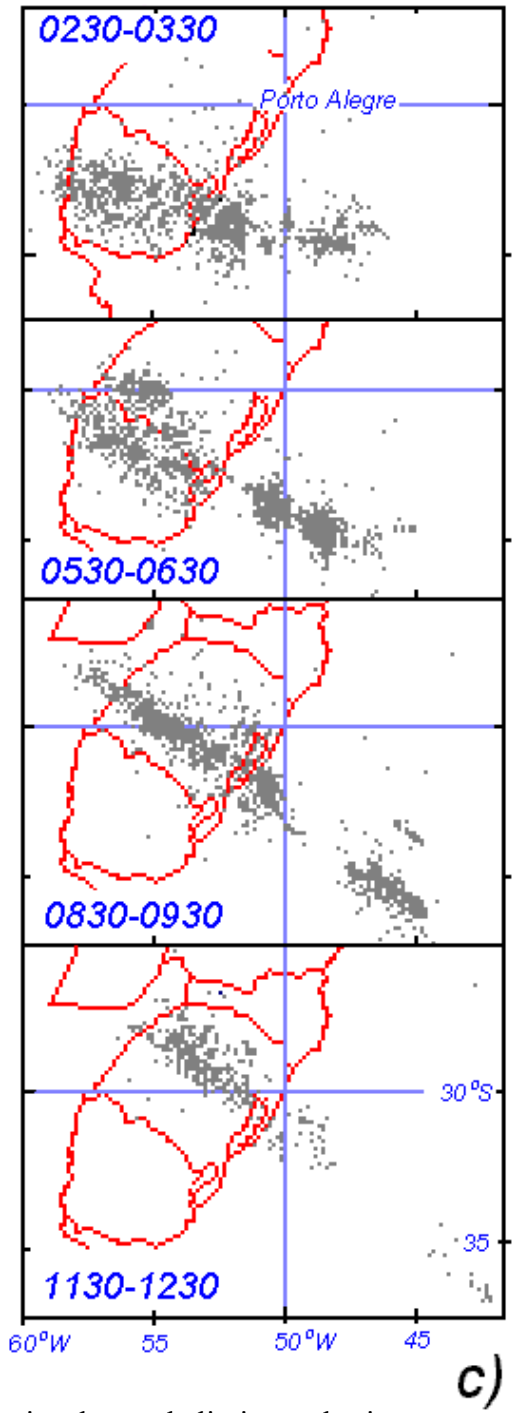

Figura 1 - Exemplo de monitoramento da frente na região do estudo. a) região de estudo limita-se, basicamente, por raios de abrangência de 240 e $480 \mathrm{~km}$ do radar da Universidade de Pelotas (círculos). Contornos com \% delineiam a eficiência de detecção na rede integrada de detecção das descargas atmosféricas (Fonte: FURNAS Centrais Elétricas). Sensores de raios TOA operados por CEMIG, FURNAS e SIMEPAR são marcados com pequenos círculos, quadrados e triângulos, respectivamente, os sensores DF em cruzes; b) integração de dados de raios com imagem de GOES-8 em infravermelho. Descargas à terra são acumuladas durante 30 minutos próximos à 0900 GMT no dia 30/07/99 e são sobrepostas (preto) para nebulosidade frontal com temperaturas menores $-3^{\circ} \mathrm{C}$ (escala de cinza); c) movimento da mesma frente em seqüências de campos de descargas acumulados durante 1 hora.

Figure 1 - Example of front monitoring in the region of study. a) region of study, limited basically, with Pelotas University radar ranges (circles) of $240 \mathrm{~km}$ and $480 \mathrm{~km}$. Contours with \% detection efficiency of Brazilian cloud-to-ground lightning network (Source: FURNAS Centrais Elétricas). TOA sensors are demarcated with small circles, squares and triangles, and direction finder with crests. b) integration of the lightning data with infrared image of GOES-8. Cloud-to-ground flashes accumulated during 30 minutes (black) around 0900 GMT, $30 / 07 / 99$, are superimposed on frontal cloudiness with temperatures less than $-3^{\circ} \mathrm{C}$ (gray scale); c) the movement of this front in time sequence of hourly integrated cloud-to-ground data.

observação da frente que passou no oceano Atlântico, nas latitudes de São Paulo e do Rio de Janeiro, na zona de baixa eficiência do RIDAT;

c) para comparação foram analisados, também, os casos 1 e 2 da linha de instabilidade tropical localizada na mesma latitude da última frente, mas quase inteiramente sobre o continente na zona de maior eficiência do RIDAT.
A quantidade total de raios $\mathrm{CG}$ registrados durante o período de 543 horas foi superior à 445000 , variando de menos 1 mil à mais de 100 mil por dia (Tabela 1). Observou-se que a polaridade e o valor da corrente de descarga não foram determinadas em $48 \%$ das descargas. Os raios sem registro de corrente foram associados com sistemas distantes da região onde se localizam os detetores de descarga, porque 


\begin{tabular}{|c|c|c|c|c|c|c|c|c|}
\hline \multirow[t]{3}{*}{$\mathrm{N}$} & \multirow{3}{*}{$\begin{array}{l}\text { Data } \\
1999\end{array}$} & \multirow{3}{*}{$\begin{array}{l}\text { Hora } \\
\text { GMT }\end{array}$} & \multicolumn{3}{|c|}{ Descargas à terra, CG } & \multirow{3}{*}{$\begin{array}{c}\text { corrente } \\
\text { registrada } \\
\text { vs. total } \\
\%\end{array}$} & \multirow{3}{*}{$\begin{array}{c}\text {-CG vs. } \\
\text { com } \\
\text { corrente } \\
\%\end{array}$} & \multirow{3}{*}{ SITUAÇÄODE GRANDE ESCALA } \\
\hline & & & \multirow[t]{2}{*}{$\begin{array}{l}\text { soma } \\
\text { total }\end{array}$} & \multicolumn{2}{|c|}{$\begin{array}{c}\text { com corrente } \\
\text { registrada }\end{array}$} & & & \\
\hline & & & & $-\mathrm{CG}$ & $+\mathrm{CG}$ & & & \\
\hline 1 & 2303 & $00-24$ & 25233 & 22092 & 3141 & 100,0 & 87,55 & Linha de instab, MG-RJ- Oceano (O) \\
\hline 2 & 2403 & $00-24$ & 20176 & 17331 & 2845 & 100,0 & 85,9 & Linha de instabilidade, GO-RJ-O \\
\hline 3 & 2805 & $15-24$ & 7421 & 294 & 71 & 4,9 & 80,5 & CC no RS, vórtice ciclônico \\
\hline 4 & 0906 & $00-24$ & 9390 & 767 & 767 & 16,3 & 50,0 & CC na Argentina, frente norte RS \\
\hline 5 & 2906 & $13-17$ & 12 & 11 & 0 & 91,7 & 100,0 & Frente fria em SClbaixa oceano \\
\hline 6 & 1407 & $00-24$ & 15638 & 503 & 239 & 4,7 & 67,8 & Frente fria no $\mathrm{RS}$ \\
\hline 7 & 1607 & $01-24$ & 2760 & 169 & 146 & 11,4 & 53,6 & Frente, Complexo Convectivo (CC) \\
\hline 8 & 1907 & $19-24$ & 328 & 72 & 85 & 47,9 & 45,8 & Frente quente no RS \\
\hline 9 & 2007 & $00-21$ & 4650 & 1764 & 652 & 52,0 & 73,0 & Frontogênese, tempestades local \\
\hline 10 & 2607 & $13-24$ & 9598 & 4237 & 628 & 50,7 & 87,0 & Ciclogênese local, setor quente \\
\hline 11 & 2707 & $00-24$ & 23268 & 9695 & 3639 & 57,0 & 72,7 & Frontogênese, cavado NW-SE \\
\hline 12 & 2807 & $00-24$ & 14436 & 1229 & 224 & 11,5 & 84,5 & Frente no oceano, litoral SPIRT \\
\hline 13 & 2907 & $00-24$ & 10918 & 58 & 18 & 7,0 & 76,0 & Frente $20^{\circ} \mathrm{S}$ em $\mathrm{O}$ e frente $35^{\circ} \mathrm{S}$ \\
\hline 14 & 3007 & $00-24$ & 17645 & 1418 & 1178 & 14,7 & 54,6 & Passagem frente fria através RS \\
\hline 15 & 0608 & $06-24$ & 347 & 125 & 16 & 40,6 & 88,7 & Zona instabilidade pré-frontal \\
\hline 16 & 1208 & $06-24$ & 864 & 64 & 71 & 15,6 & 47,4 & Zona instabilidade pré-frontal \\
\hline 17 & 2508 & $00-24$ & 1361 & 12 & 11 & 1,7 & 52,0 & Frente fria no RS \\
\hline 18 & 2708 & $03-24$ & 2816 & 1614 & 1002 & 92,8 & 61,7 & Ciclogênese local no RS \\
\hline 19 & 3008 & $00-24$ & 5821 & 1 & 0 & 0,0 & 100,0 & Frente quase-estacionária, Uruguai \\
\hline 20 & 3108 & $00-24$ & 15759 & 99 & 622 & 4,5 & 13,7 & Setor quente . frente, ciclogênese, \\
\hline 21 & 0109 & $00-18$ & 13588 & 402 & 5178 & 41,0 & 7,2 & Ciclogênese local no R.S \\
\hline 22 & 0209 & $13-24$ & 7224 & 126 & 2712 & 39,3 & 4,4 & Ciclogênese local no RS \\
\hline 23 & 0309 & $00-24$ & 21326 & 249 & 7685 & 37,2 & 3,1 & Ciclogênese local no RS \\
\hline 24 & 0709 & $00-24$ & 38879 & 67 & 1647 & 4,4 & 3,9 & Ciclog. local setor quente, frente fria \\
\hline 25 & 0809 & $00-24$ & 98383 & 8149 & 47813 & 56,9 & 14,6 & Ciclogênese local, frente fria SC \\
\hline 26 & 1309 & $00-24$ & 9815 & 2525 & 3067 & 57,0 & 45,0 & Vórtice ciclônico sul RS, frente \\
\hline 27 & 1409 & $00-24$ & 68244 & 22616 & 35014 & 84,4 & 39,2 & Ciclogênese e frontogênese \\
\hline & ma & \#543 & $\begin{array}{c}\# 445900 \\
100 \%\end{array}$ & $\begin{array}{l}\text { 495689 } \\
21.4 \%\end{array}$ & $\begin{array}{l}\# 118471 \\
26,6 \%\end{array}$ & $\frac{42,4 \%}{48 \%}$ & $\frac{61 \%}{44.7 \%}$ & $\begin{array}{l}\text { Valor Médio Simples, } \% \\
\text { Valor Médio Ponderado, } \%\end{array}$ \\
\hline
\end{tabular}

Tabela 1 - Situação de grande escala vs. atividade elétrica durante observação.

Table 1 - Large scale situation vs. electrical activity during observation.

a amplitude do sinal de descarga de retorno nos receptores foi pequena demais.

As descargas que ocorrem eventualmente no tempo e no espaço são dados pontuais e, em contraste com as características atmosféricas contínuas (e.g. temperatura, velocidade do vento, índice de refração, etc.), só podem ser apresentadas em campos acumulados, ou seja, é necessário obter uma acumulação temporal das posições de descargas, denominado Campo Acumulado das Descargas (CAD). Considerando-se o caráter experimental do trabalho, para rápida leitura dos registros e visualização dos CAD's na imagem de vídeo e seu arquivamento posterior, em formato "bmp", foram elaboradas as rotinas na linguagem BASIC, de fácil modificação. Variando o tempo de acumulação, os CAD's foram examinados em função de seu possível uso em: a) determinação do movimento dos sistemas de precipitação de escala meso a (300-600 km e tempo de vida de mais de 6 horas); b) análise da situação sinótica durante a ciclogênese, comparando-se as estruturas observadas no CAD e as mesmas nas imagens de satélite GOES-8. Levando-se em conta que as imagens de GOES-8, nestas latitudes, têm resolução em torno de $10-15 \mathrm{~km}$, com intervalo mínimo de 30 minutos entre as imagens, a mesma resolução foi usada na obtenção do CAD para acompanhamento dos sistemas de escala meso a (Fig. 1b). Na figura 2 são mostrados os CAD com integração de raios em pixels de $\cong 0,14^{0}$ de longitude $\times 0,13^{0}$ de latitude (i.é. $14,5 \times 14,5 \mathrm{~km}$ na latitude $25^{\circ} \mathrm{S}$ ) e codificação de tempo em cores (o método de visualização das descargas, como na figura 2, praticado a mais de 15 anos, vide Browning, 1989).

Além dos sistemas da escala- $\alpha$, é estudada a viabilidade de visualização em CAD das estruturas dos sistemas de precipitação de escala meso $\beta$ no RS, cujo tempo de evolução é menor do que 6 horas 
e tamanho típico $\leq 300 \mathrm{~km}$. Baseando-se na presença da refletividade dos núcleos convectivos $(\mathrm{Z})$, seu alinhamento no indicador plano do radar (PPI), e nas alturas de ecos de radar em seções verticais (RHI), que ocorrem no interior de um raio de $240 \mathrm{~km}$ do radar, a organização da precipitação e da intensidade do sistema foram classificados em seis padrões. Para comparação visual das estruturas dos sistemas dos campo de refletividade e raios, os CAD's foram construídos com resolução de $3 \times 3 \mathrm{~km}$, intermediária entre a resolução espacial das imagens em $240 \mathrm{~km}$ $(2 \times 2 \mathrm{~km})$, e em $480 \mathrm{~km}(4 \times 4 \mathrm{~km})$. Os campos acumulados em 15 minutos antes e após o horário da imagem do radar foram superpostos na mesma projeção (Fig. 5).

\section{SISTEMAS DE ESCALA- $\alpha$}

A quantidade de raios produzidos nos 16 sistemas frontais examinados variaram muito, em torno de 3 raios por hora no caso da frente fria em dissipação (caso 5) até 970 por hora no caso 11. Em média, durante uma hora os sistemas frontais produziram 415 raios. Dependendo da localização geográfica dos sistemas frontais em relação à rede de detetores, a razão percentual da quantidade das $\mathrm{CG}$, com corrente registrada, varia de quase $100 \%$ na fronteira entre estados Santa Catarina e Rio Grande do Sul (RS) até $0 \%$ no Sul do RS (Tabela 1 ). Da tabela 1 pode-se ver que em 28,29 e $30 / 07$, quando os sistemas frontais foram localizados fora da zona de probabilidade $20 \%$ de detecção da rede, somente 4 a $15 \%$ das correntes foram registradas.

Quase metade dos raios da amostra (Tabela 1) foi registrada nos 9 sistemas associados à ciclogênese local no RS. A taxa horária média nesses sistemas foi de 1656 descargas por hora, ou seja, 4 vezes maior do que a dos sistemas transientes, e a porcentagem dos raios com corrente registrada aumentou até 40$50 \%$.

De acordo com a polaridade, os raios negativos apresentam $44,7 \%$ dos raios com polaridade registrada (Tabela 1), divergindo dos estudos da climatologia de raios em outras regiões onde os raios negativos apresentam-se em torno de $80-90 \%$ da amostra total (Gin et al., 2000; Hodanish et al., 1997; Orville \& Silver, 1997). Isto poderia ser explicado pelo fato de que as amplitudes das correntes de raios positivos são maiores do que as de raios negativos nessa amostra (Abdoulaev et. al., 2000) e, consequentemente, a proporção entre os raios positivos e negativos deve crescer com a distância dos detectores. Outro fato é que estação upode contribuir significativamente na observação de raios positivos. As estatísticas de polaridade de raios (Mazin \& Khargian, 1989; Orville \& Silver, 1997) mostram que geralmente os raios que levam à terra carga positiva são mais frequentes no inverno. Orville \& Silver (1997), nos EUA, observaram um aumento da porcentagem das descargas positivas de até $25 \%$ em dezembro, em contraste com os $3 \%$ observados em agosto. A possibilidade de detecção dos sistemas que produzem os raios de certa polaridade e corrente, além da variação sazonal, é associada, naturalmente, com o tipo do sistema particular observado. Na tabela 1 pode-se ver que na linha de instabilidade tropical (casos 1 e 2), observada dentro da RIDAT, a taxa horária foi quase 1 mil descargas por hora. A polaridade de raios foi registrada em $100 \%$, pois a linha ocorreu na região de probabilidade estimada de detecção de raio $>95 \%$. Os $85-87 \%$ dos raios nessa linha foram de polaridade negativa. A mesma porcentagem de raios negativos observou-se na área de atuação da frente fria (caso 12) que ocorreu na mesma latitude que a linha de instabilidade, embora sobre o oceano, longe da rede de detetores, produzindo 600 descargas por hora com pequena quantidade, de $11 \%$, das correntes registadas. Por outro lado, durante o caso 27 com ciclogênese e frentes atuando na área, com mais de $84 \%$ de polaridades registadas, $60 \%$ dos raios foram positivos. Considerando-se que, nas áreas de ciclogênese, em $70 \%$ dos casos (Tabela 1) a porcentagem de descargas positivas entre os raios com a polaridade registrada foi maior que $50 \%$, pode-se supor que a nebulosidade ciclonal, com sua complexa estrutura e dinâmica, é mais propícia à geração de altas taxas de raios, inclusive os de polaridade positiva e grande amplitude. Este fato também comprova a afirmação anterior de que as descargas à terra concentram-se no centro do ciclone (Ludlam, 1980) e estudos mais recentes (Reap, 1993; McGorman et al., 1993) que revelam o papel da situação meteorológica de grande escala na atividade elétrica. Analisando as cartas 
sinóticas de 831 dias, no período de 1987-1990, Reap (1993) observou que nos fluxos de SE e SW ocorreram $60 \%$ dos CG registrados na Florida. MacGorman et al. (1993) classificaram 12 tipos de situações sinóticas em Oklahoma de acordo com a temperatura do ponto de orvalho na superfície e a direção do vento a $500 \mathrm{hPa}$, e verificaram que somente 3 tipos de circulação são responsáveis por $75 \%$ das descargas à terra no ano.

\section{FRENTES TRANSIENTES}

A nebulosidade das frentes com deslocamento rápido, geralmente têm uma forma com característica linear ou de arco não acentuado, e facilmente se distingue nas imagens de satélite e nos campos acumulados de descargas atmosféricas (CAD), mesmo em casos da taxa baixa de raios. A figura $1 \mathrm{~b}$ mostra o CAD de 0845 à 0915 GMT, sobreposto as nuvens frontais às 0900 horas do dia 30 de julho de 1999, observados nas imagens GOES-8, em infravermelho. Observa-se que, sobre o continente, o CAD apresenta um faixa relativamente estreita que se localiza na parte $\mathrm{SW}$ da nebulosidade com temperaturas do topo abaixo de $-3^{\circ} \mathrm{C}$, e largura de $350-450 \mathrm{~km}$. Embora a forma de cunha do CAD, em geral, segue a forma triangular das nuvens de temperaturas com $<-20^{\circ} \mathrm{C}$, a nebulosidade mais alta é distribuída uniformemente na direção de SE para $\mathrm{NW}$, enquanto as descargas aglomeram-se em centros distanciados por cerca de $100 \mathrm{~km}$ um do outro. As aglomerações de descargas é uma característica notável dos campos acumulados durante períodos de cerca de 30 minutos. Essa propriedade do $\mathrm{CAD}$ não impede a determinação do deslocamento da frente em campos acumulados durante 1 hora. Na Fig. 1c mostra-se o acompanhamento da frente do dia 30/ 07/99, durante mais de 10 horas seguidas. As descargas associadas à frente fria entre 0230 e 0330 GMT localizaram-se no centro do Uruguai e oceano próximo ao litoral. O CAD apresenta uma faixa ondulada, mostrando a frente fria com uma faixa côncava para cima e a seção frontal quente côncava para baixo. Posteriormente, durante o deslocamento pelo oceano, essas partes da frente deram origem ao ciclone cuja nebulosidade produziu as descargas até a latitude de $40^{\circ} \mathrm{W}$ (vide Fig. 1b). Nos CAD's, entre
0530 e 0630 GMT, a zona frontal fria se lineariza, mudando a orientação de W-SW para SW. Como pode ser determinado a partir do deslocamento da parte dianteira do $C A D$, a frente teve a velocidade média de cerca de $70 \mathrm{~km} / \mathrm{h}$ e, por volta de $1200 \mathrm{GMT}$, se encontrava sobre Porto Alegre, RS.

Observou-se que na determinação de movimento das frentes em regime operacional, podem ser úteis os CAD's de acumulação de 2 horas, sobrepostos um sobre o outro numa mesma imagem de vídeo, com código temporal de cores apropriado, tal como é mostrado na figura 2. A análise desses CAD's permite observar o movimento de sistemas tanto no continente (e.g. no dia 30/07/99) como no oceano. Por exemplo, da figura 2 é fácil acompanhar a posição da frente fria que se observou sobre o oceano de 2200 GMT do dia 27/07/99 (ponto F0), na latitude de Paranaguá, PR, até as 1200 GMT do dia 29/07/99, na altura de Vitória, ES (ponto F4). No seu deslocamento foi conservada a orientação de NE à SW. Mesmo com a diminuição da atividade elétrica no dia 29 , a posição da frente pode ser atribuída à parte dianteira da superposição horária no CAD (e.g. entre 0200 à 0600 GMT no dia 29/07/ 99, Fig. 2). As frentes com raios sem polaridade determinada foram localizadas em CAD a centenas de quilômetros a leste do continente, no Oceano Atlântico (caso 12), no sul do Rio Grande do Sul e no Uruguai até a foz do Rio La Plata (casos 19-20), no Paraguai, no norte e nordeste da Argentina. Assim, a ausência da corrente registrada, e a diminuição da precisão espacial na detecção de raios não é obstáculo para o acompanhamento horário dos sistemas frontais lineares.

\section{SISTEMAS DURANTE CICLOGÊNESE LOCAL}

Observou-se que os casos do ciclogênese local (e.g. casos 23-24) são os mais complexos para o acompanhamento da evolução do sistema nos CAD de 1-2 horas de acumulação, principalmente por causa do desenvolvimento de nebulosidade nova que geralmente ocorre sobre mesmo local. Quando isto ocorre os raios acumulados nos instantes precedentes são disfarçados por CAD’s posteriores. Além disso, as formas do CAD nesses casos são mais irregulares 


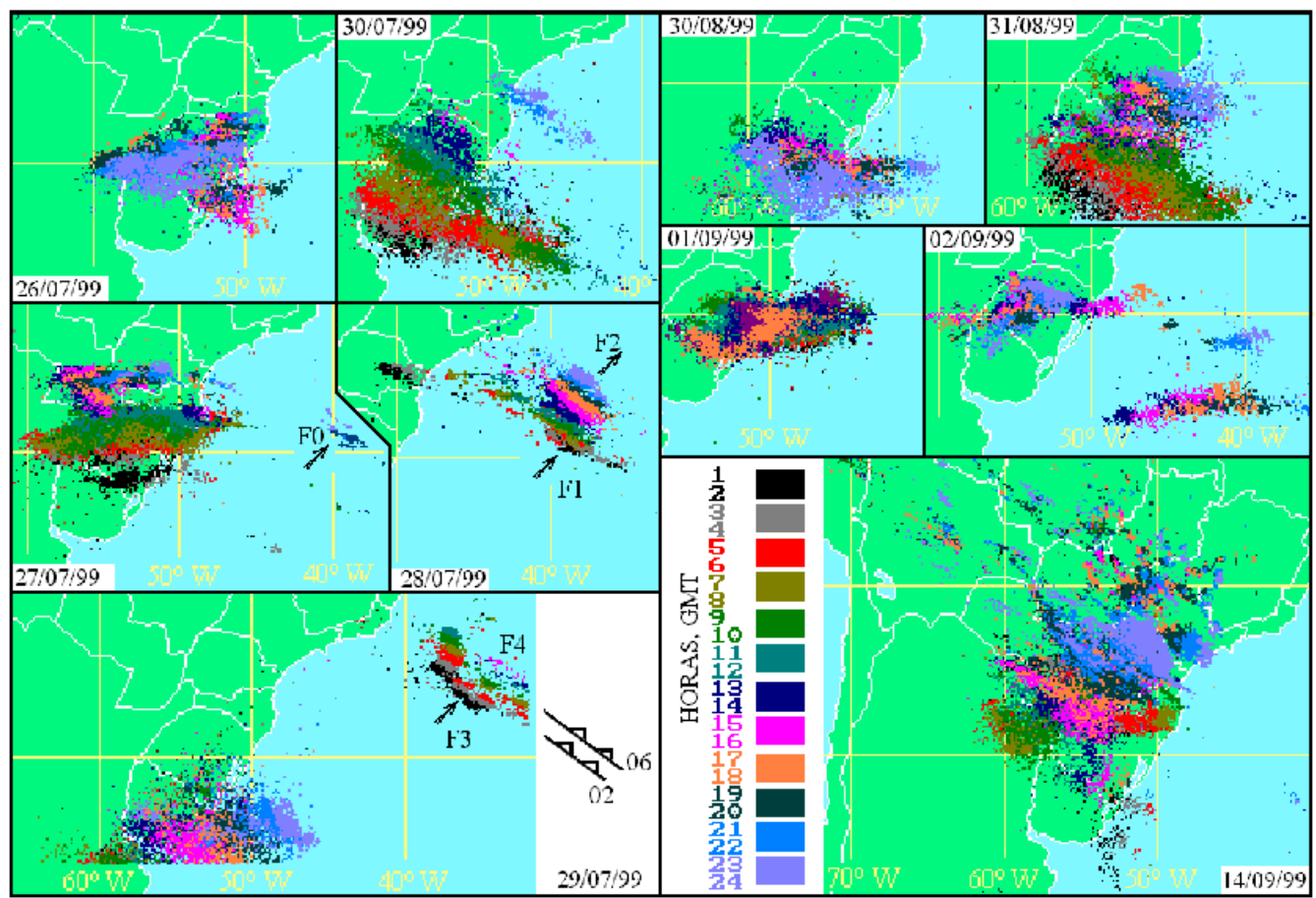

Figura 2 - Campos Acumulados das Descargas (CAD) durante ciclogênese local, dia 26/07/99, e desenvolvimento posterior da frente que move para interior do continente, no dia 27/07/99, precedendo a passagem da frente fria rápida nos dias 28/07/99 à 30/07/99. Linha da frente oceânica é marcada nas posições de F0 a F4 e é facilmente determinada durante os dias 27-29/07/99 (e.g. entre 0200 e 0600 GMT, 29/07/99). CAD da frente estacionária no dia 30/08/99; CAD durante ciclogênese frontal (31/08/99) e local (01/09/99-02/09/99, 14/09/99). O tempo é codificado em cores.

Figure 2 - Time Integrated Lightning Fields (TILF) during local cyclogenesis, 26/07/99, and in a developing front, 27/07/99 that moves towards inland and preceding the passage of a fast cold front from 28/07/99 to 30/07/99. The oceanic front line is given by the position of F0 to F4 and it is easily determined during days 27-29/07/99 (e.g. between 0200 and 0600 GMT, 29/07/99). TILF of a stationary front on 30/08/ 99; TILF during frontal cyclogenesis (31/08/99) and local (01/09/99-02/09/99, 14/09/99). Time is color coded.

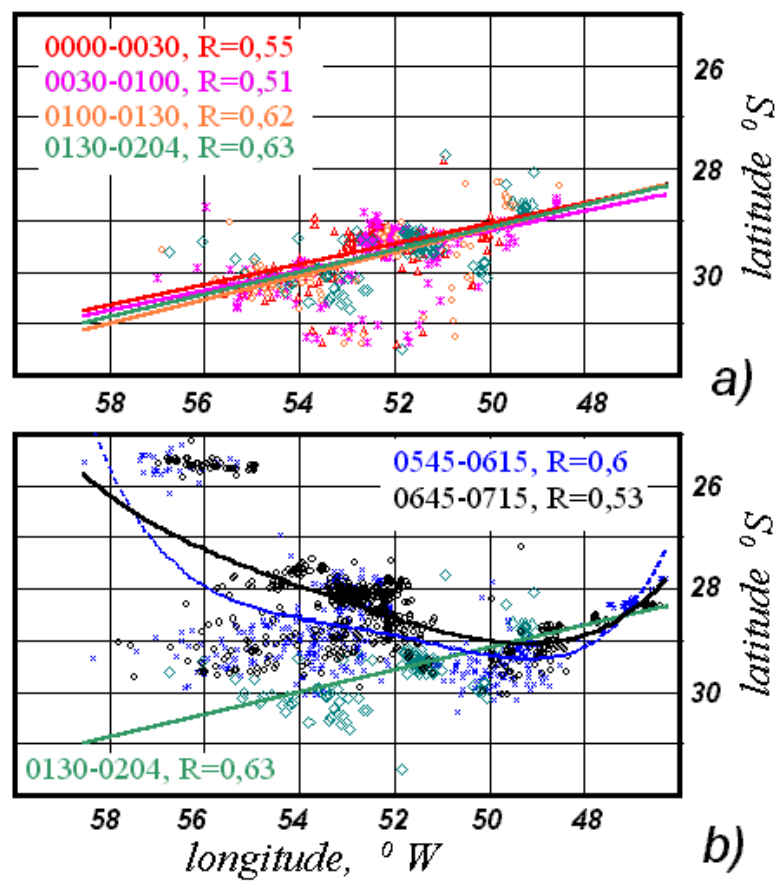

Figura 3 - Ajuste da posição da linha frontal pelo método dos quadrados mínimos usando acumulação dos raios durante 30 minutos: (a) 0000 0204 GMT de 27/07/99, estágio inicial; (b) estagio de maturidade do sistema 0545-0715 GMT com movimento do sistema ao norte. R são os coeficientes de correlação.

Figure 3 - Frontal line position are adjusted by minimum square method using accumulated lightning data during 30 minutes (a) 0000-0204 GMT, 27/07/99, initial stage, (b) system at maturity stage, 0545-0715 GMT, with northward movement of the system. $R$ stands for the correlation coefficient. 
do que aqueles referentes às frentes (Fig. 2). Por exemplo, a interpretação dos CAD's durante a ciclogênese nos dias 26 e 27/07/99 é mais complexa, em contraste com a análise dos CAD's referentes às frentes frias rápidas dos dias 28, 29 e 30/07/99.

\section{a) delineação das linhas frontais}

Se houver alta taxa temporal de raio, é possível obter uma informação sobre a posição e a forma da frente, analisando-se os campos acumulados nos períodos menores do que 1 hora. Por exemplo, foi possível delinear as frentes durante a ciclogênese local no dia 27/07/99, por um período de 6 horas (Fig. 3) de 0000 à 0600 GMT. Nestes instantes, quando a atividade elétrica ao norte do RS alcançou valores da ordem de 100 à 200 descargas em 5 minutos, em intervalos curtos de 15-30 min pode-se obter uma amostragem de posições de raios suficiente para ajustar da posição frontal por meio do método dos

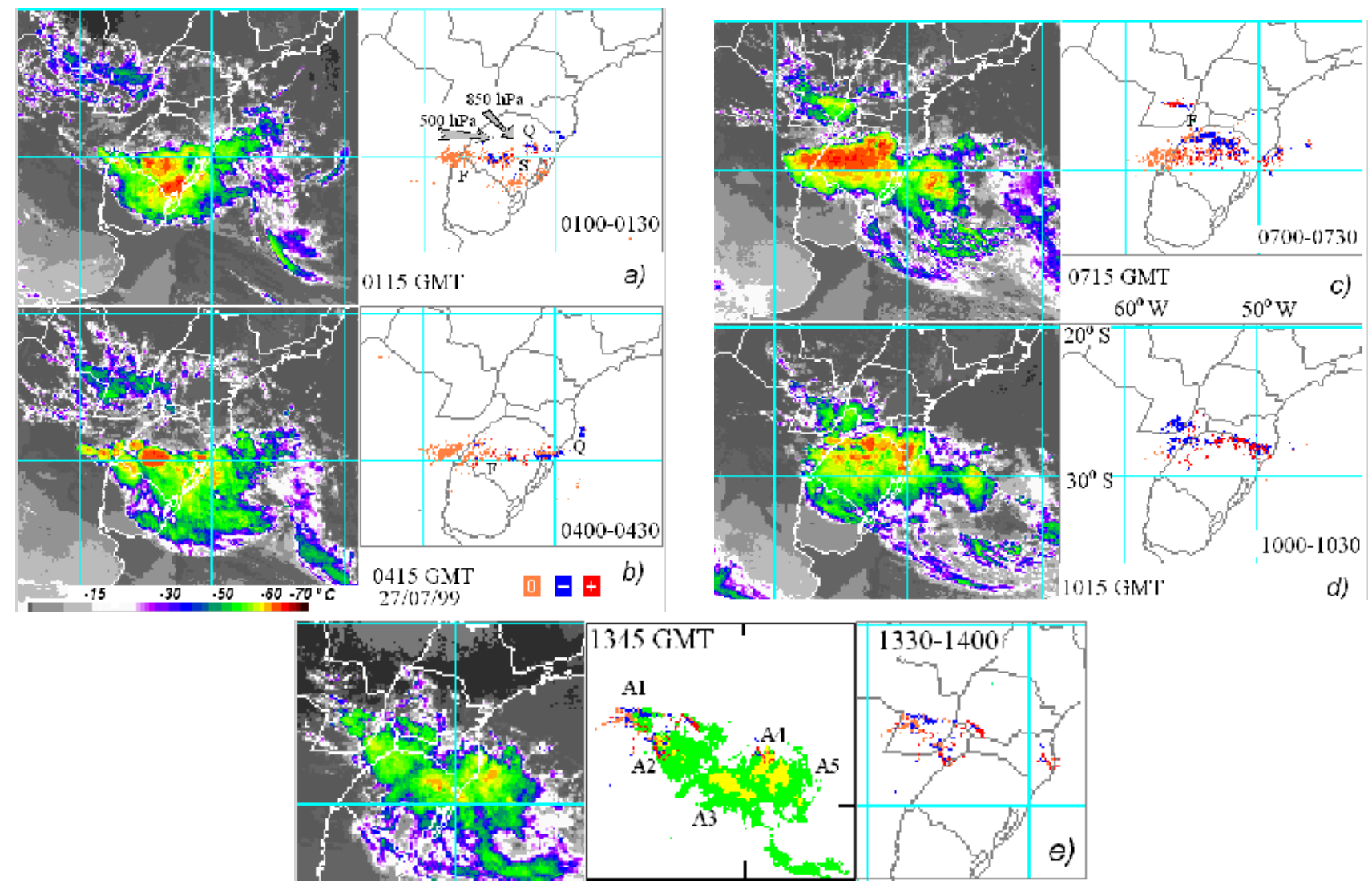

Figura 4 - Evolução do sistema no dia 27/07/99, CAD vs. GOES-8: a) e b) são estágios iniciais com seções quente (Q) e fria (F). Note que a região $\mathrm{S}$ (a) dissipa totalmente durante três horas entre as imagens; $\mathbf{c}, \mathbf{d}$ ) maturidade do sistema com desenvolvimento da linha pré-frontal F no CAD (c) e sua pós-visualização na imagem de satélite (d); e) sistema dissipa; aglomerações A3 e A5 não produzem descargas à terra.

Figure 4 - System evolution on 27/07/99: TILF vs. GOES-8: a) e b) are initial stages with warm $(Q)$ and cold $(F)$ sections. The region $S$ (a) disappears totally during three hours between images; $\boldsymbol{c}, \boldsymbol{d})$ mature system with a development of a pre-frontal line $F$ in the TILF (c) and with post-visualization in the satellite image $(d), e)$ disperse system, ensembles A3 and A5 do not produce cloud-to-ground lightning. 
diferente do que nos instantes iniciais, quando as posições dos raios, distribuindo-se mais regularmente, foram alinhados numa faixa estreita de sudoeste à nordeste. Em seguida, será apresentada uma análise comparativa dos estágios da ciclogênese ocorrida neste dia, visualizado-se em CAD e imagens de satélite correspondentes.

\section{b) seções frontais e estágios da ciclogênese em CAD e imagens de satélite.}

Nesse parágrafo será demonstrado que nos casos em que os CAD's perdem a forma linear, e a direção do deslocamento do sistema não é óbvia, sendo necessária uma análise detalhada das estruturas formadas por descargas na seqüência temporal de CAD, e de nebulosidade na imagem de satélite. Também será discutido como a visualização da polaridade dos raios permite a interpretação mais segura do tipo da seção frontal (quente, ou fria), do que aquela que é baseada na análise de temperaturas dos topos das nuvens.

Como foi observado nos CAD's na figura 2 e figura 3, a evolução do sistema de nuvens no dia 27/ 07/99 foi bastante complexa. Na imagem em infravermelho do GOES-8 no horário de 0115 GMT e no $\mathrm{CAD}$ feito no intervalo de 30 minutos próximo ao instante da imagem de satélite (Fig. 4a), pode-se ver que a nebulosidade composta das aglomerações delineadas pela temperatura $<-50^{\circ} \mathrm{C}$ e os pontos de raios formam uma concavidade. A parte norte desta concavidade tem orientação quase perpendicular ao vento nos níveis baixos da atmosfera $(900-850 \mathrm{hPa})$ que traz ar quente e úmido da região do Chaco e Missiones, enquanto a parte sul da concavidade tem orientação latitudinal, quase paralela ao vento, nos níveis médios $(500 \mathrm{hPa})$. Considerando-se que a parte norte do sistema tem movimento mais lento que à translação passiva com o vento na troposfera média (a velocidade de translação estimada de dados de radar às $21 \mathrm{GMT} 260799$ foi $70 \mathrm{~km} / \mathrm{h}$ e $290^{\circ}$ ), podese dizer que aparecem formações de Cbs no sentido oposto à componente de translação, normal ao eixo da linha, ou seja, a propagação normal negativa (Abdoulaev \& Lenskaia, 1998). As linhas de convecção com propagação negativa têm região estratiforme advectada adiante da região convectiva, e geralmente, são encontradas na seção frontal quente (Q - na Fig. 4). Na imagem de satélite, entretanto, pode-se ver que os "aglomerados" S a sotavento da frente, Q, são transladados sobre a Lagoa dos Patos para o oceano e, dificilmente, podem ser interpretados como regiões estratiformes, tendo grandes áreas dos topos das nuvens com temperaturas abaixo de $-50^{\circ} \mathrm{C}$.

A análise da distribuição de raios (Fig. 4a) mostra que os vastos aglomerados de nuvens altas $\mathrm{S}$ têm pouca atividade convectiva. Este fato, junto com a evidência de que a sudeste da linha $Q$ prevalecem os raios positivos, permite supor que a convecção nos $\mathrm{S}$ está dissipando, pois as descargas positivas são mais freqüentes na região estratiforme que acompanha as linhas de convecção (vide e.g. Rutledge \& MacGorman, 1988). Realmente, 3 horas depois (Fig. 4b) as nuvens altas e os raios nestas regiões desaparecem, a orientação do sistema começa se transformar em latitudinal, com convexidade na parte oeste do sistema. Essas partes do sistema (F na Fig. 4a e Fig. 4b), quase paralelas ao vento em $500 \mathrm{hPa}$, visivelmente deslocam-se lentamente, significando que há forte propagação negativa paralela, o que compensa uma translação de NW-W para E-ES com o vento na troposfera média. A forma convexa que corresponde à seção fria da frente, com o passar do tempo (Fig. 4c), fica mais saliente. Ao norte desta seção, os raios negativos formam um notável arco, $\mathrm{F}$, que corresponde a uma linha de instabilidade préfrontal, marcada na imagem de satélite somente pela fina linha de $\mathrm{Cb}$. Pode-se observar que as nuvens dessa linha, tendo alturas que não ultrapassam a $-50^{\circ}$ $\mathrm{C}$, são muito mais ativas eletricamente em comparação com a vasta área de nebulosidade frontal mais alta que produz quantidades moderadas de raios positivos e raios sem registro de corrente. $\mathrm{O}$ surgimento do arco F no CAD, Fig. 4c, de certo modo, "precede por fase" a imagem de satélite, Fig. 4d, onde é evidente a coexistência das duas bandas frontais. Uma linha frontal inicial começa a ser mais difusa com o deslocamento da atividade dos raios negativos para oeste e os positivos para leste. Observações com radar mostram que a transformação das linhas da convecção severa, durante intervalos superiores a 1 hora, em alguns focos de convecção menos organizados, freqüentemente significa que o sistema começa a dissipar. É importante notar que, elaborando 
os CAD's de maior resolução (e.g. CAD na Fig. 3 b), é possível ver que os núcleos de atividade elétrica começam perder sua organização linear em torno de 0600 horas, bem antes que seria possível deduzir isso na análise da imagem de satélite das 0715 horas (Fig. $4 c)$. Após o meio dia a dissipação da zona frontal começa, também, ser vista na imagem de satélite (Fig. 4e). Nestes momentos, como nos instantes anteriores, é notável a relação complexa entre atividade elétrica e as alturas de nuvens. As aglomerações A3 e A5, com topos de nuvens elevados nas imagens em infravermelho, por exemplo, não produzem raios, enquanto se observam raios nas aglomerações A1, A2, A4. Roohr \& Vonder Haar (1994), explorando os dados de descargas para acompanhamento de frente fria e ciclone tropical, demonstraram que não existe uma relação óbvia entre topos de nuvens nas imagens em infravermelho e a taxa de raios. O problema da variação de taxa de raios em relação ao tipo e a altura das nuvens será discutido abaixo.

\section{SISTEMAS DE ESCALA $\beta$}

\section{a) classificação dos sistemas observados}

Baseando-se na análise de eco de radar no raio de $240 \mathrm{~km}$, em Pelotas, RS, foi determinado que, durante as passagens das frentes e ciclogenêse local, a severidade e organização dos 37 sistemas de mesoescala de precipitação (SMP) da escala b que produzem os raios, variam de um caso para outro (Tabela 2). Particularmente, foram observados os seguintes tipos dos SMP (cujos termos e definições são os mesmos mostrados em Abdoulaev, et al., 1998):

(a) 4 Linhas de Convecção Severa (LCS ou L1), que são os segmentos longos ( $>200 \mathrm{~km})$ e estreitos das células convectivas com a $Z>55$ $\mathrm{dBZ}$, acompanhados ou não pela região das precipitações estratiformes. Três casos de LCS foram observados durante a ciclogênese local, na área coberta pelo raio de $240 \mathrm{~km}$, sobre a região de Pelotas; uma LCS foi associada com a frente semi-estacionária no dia 30/08/99, 350 $400 \mathrm{~km}$ ao sul do radar;

(b) 4 Sistemas Não Lineares de Convecção Severa (NLCS ou N1) da mesma intensidade de L1 representando, entretanto, um agrupamento desorganizado das tempestades severas e das áreas de precipitação estratiforme surgidos durante a ciclogênese local, sendo que os $3 \mathrm{~N} 1$ foram observados no mesmo dia de L1;

(c) 10 Bandas de Precipitação (classe L2) e 3 Sistemas de Chuvas Esparsas (classe N2) identificados como sistemas semelhantes na organização de L1 e N1, mas com células de refletividade menor de $55 \mathrm{dBZ}$, geralmente em torno de $35-45 \mathrm{dBZ}$; as L2 e os $\mathrm{N} 2$ foram observados em associação com as frentes e zonas frontais (8 casos) bem como durante a ciclogênese ( 4 casos locais e 1 em associação com a passagem do complexo convectivo);

(d) 7 sistemas de precipitação estratiforme com elementos de refletividade elevada alinhados em bandas paralelas (sistemas Stratiformes Lineares - SL) e 7 sistemas com tais elementos distribuídos caoticamente (stratiformes não lineares SN); os 6 SL foram observados nas frentes quente e fria, enquanto $5 \mathrm{SN}$ foram observados em outras condições de grande escala.

As tempestades convectivas ordinárias de curta duração, $<1$ hora, são designadas por "O". Em geral, observou-se a prevalência dos sistemas convectivos organizados linearmente (as linhas de $\mathrm{Cb}$ foram observadas em 13 de 22 casos), o que corresponde à variação sazonal na estrutura de sistemas de precipitação no Rio Grande do Sul (Abdoulaev et al., 1998). Nota-se que somente os 2 dos $7 \mathrm{SN}$ foram observados separadamente de L2 ou SL.

Da tabela 2 pode-se ver, num círculo de $480 \mathrm{~km}$ do radar, durante o período total de observação com radar, foi registrado 40851 raios, incluindo 5590 raios de polaridade positiva e 3083 raios de polaridade negativa. A polaridade positiva predominante das correntes registradas foi observada em 15 casos nos 22 dias. A maioria desse casos foi associado com sistemas estratiformes SL e SN, além dos sistemas $\mathrm{L} 1$ e N1 acompanhados por regiões estratiformes, $\mathrm{e}$ a quantidade de raios positivos $(+\mathrm{CG})$ foi maior de 2 à 12 vêzes do que os negativos (-CG). Uma quantidade significativa de-CG foi observada somente em dois dias (casos 3 e 10), sendo que $2 / 3$ de todas as descargas negativas foram registradas no dia $26 /$ 
07/99, com convecção severa não linear. A taxa horária média (número do $\mathrm{GC} /$ hora), de todos os sistemas num raio de radar de $480 \mathrm{~km}$, é de 196 descargas por hora, de 78 descargas por hora num raio de $240 \mathrm{~km}$, alcançando assim densidade média de raios por quilômetro quadrado de $2,7 \times 10^{-4}$, e $4,3 \times 10^{-4}$ descargas $\mathrm{x}\left(\mathrm{h} \mathrm{km}^{2}\right)^{-1}$, respetivamente. Como era esperado, as maiores taxas foram associadas aos sistemas severos L1 e N1 que, em média, tiveram taxa num círculo de 480(240) $\mathrm{km}$ do radar de 564 (353) raios por hora, equivalente à densidade de 7,8 e $19 \times 10^{-4}$ descarga $\mathrm{x}\left(\mathrm{h} \mathrm{km}^{2}\right)^{-1}$. Considerando-se que as alturas dos sistemas severos foram superiores a $10 \mathrm{~km}$, o aumento da densidade de descargas para o círculo de diâmetro menor, significa que a maior parte do sistema severo responsável pelas descargas registradas foi observada na área de 240 quilômetros de distância próxima ao radar. Durante a observação dos sistemas estratiformes SL, SN ocorreram somente 77 (11) descarga $\mathrm{x} \mathrm{h}^{-1}$ em círculo de 480 (240) km. Nesse caso, a densidade de $1,05 \times 10^{-4}$ descarga $\times(\mathrm{h}$ $\left.\mathrm{km}^{2}\right)^{-1}$ no círculo maior supera a densidade de $0,6 \mathrm{x}$ $10^{-4}$ descarga $x\left(\mathrm{~h} \mathrm{~km}^{2}\right)^{-1}$ do círculo menor. Isso indica que durante passagem dos sistemas estratiformes na área próxima ao radar, com raio de $480 \mathrm{~km}$ há outras fontes de descargas. As tempestades ordinárias durante sua curta vida não produziram nenhum raio detectado. Embora nos casos com sistemas convectivos moderados L 2 e N2 a densidade média seja da ordem $2,5 \times 10^{-4}$ é 1,5 vezes maior que no círculo menor, observa-se aí grande variação no valor da densidade. Somente nos dias 29/07 e 12/08 todo sistema moderado foi observados no círculo de 240 $\mathrm{km}$ de raio. Os sistemas compostos de linhas de convecção moderada L2 e precipitação estratiforme $\mathrm{SN}, \mathrm{SL}(\operatorname{casos} 3,9,23,27)$ com as densidades da ordem de $2,2 \times 10^{-4}$ e $15 \times 8^{\prime} 10^{-4}$, em círculos de 480 e $240 \mathrm{~km}$, respetivamente, podem ser considerados bem definidos em dados de radar e descargas, mesmo como sistemas severos. Considerando-se que, tipicamente, os sistemas de mesoescala de precipitação ocupam uma área equivalente a área circular de raio de $300 \mathrm{~km}$, na massa de ar com que são transladados (Abdoulaev, 1995, Abdoulaev, et al., 1998), pode-se preestabelecer que os sistemas de convecção severa L1 e N1 (Z>55 dBZ) e os sistemas compostos das bandas L2, acompanhados pelas regiões estratiformes $\mathrm{SN}$, SL apresentam também os compactos fontes (tamanho $<300 \mathrm{~km}$ ) de raios. Esses sistemas surgindo durante a ciclogênese local no sul do RS podem ser discriminadas pelas densidades de 15 à 20 descarga $\mathrm{x}\left(\mathrm{h} 10000 \mathrm{~km}^{2}\right)^{-1}$ usando dados da RIDAT.

\section{b) atividade elétrica e parâmetros de radar dos sistemas}

Observou-se que a possibilidade de determinação da estrutura do sistema de precipitação aplicando o CAD depende não só da intensidade do sistema (severa, moderada, estratiforme), da distância dos detetores da RIDAT, bem como, do estágio de vida do sistema e estrutura observada na imagem de radar. Na figura 5a, por exemplo, são mostrados os PPI's do sistema severo durante o estágio não linear N1, no dia 31/08/99, e durante a sua transformação para o sistema severo linear L1 (Fig. 5b). A refletividade em N1, no instante 1058 GMT, Fig. 5a, atingiu valores de $60 \mathrm{dBZ}$, e no local do radar observou-se chuva forte, com queda de granizo de diâmetro até $1 \mathrm{~cm}$. Apesar dessa severidade, o sistema não linear produziu, durante 30 minutos, de 1045 à 1115 GMT, somente 22 descargas num raio de 240 km do radar, enquanto 4 horas depois, entre 1500 e 1530 GMT, a quantidade de descargas registradas num raio de $240 \mathrm{~km}$ do radar foi de 118, i.é., 5 vêzes maior, Neste caso, a estrutura do eco apresentou um sistema linear severo L1 (Fig. 5b). Os CAD's sobrepostos às PPI nestes instantes demonstram que apesar de a refletividade máxima ter sido menor (em torno de $55 \mathrm{dBZ}$ ), a produção de raios pelas bandas de ecos severos (B1, B2 delineados com refletividade $Z=30 \mathrm{dBZ}$, na Fig. 5b) é maior do que a das tempestades T1, T2 e T3 do sistema não linear (Fig. 5a). Além disso, no sistema N1, observou-se uma diferença significativa entre a apresentação dos seus elementos em CAD. Na zona de precipitação convectiva T2, que foi mais severa e responsável pelo granizo, por exemplo, registraram-se somente 3 raios, enquanto os outros 19 foram observados em zonas convectivas menos intensas e na região estratiforme que acompanha o sistema. Em contraste com a tempestade T2, com grande área de precipitação convectiva, e com número de descargas relativamente 
pequeno, observaram-se 7 descargas positivas na pequena tempestade T1 ocorrida a $260 \mathrm{~km}$ à NW do radar (Fig. 5a).

$\mathrm{Na}$ figura 5 (a e b), as zonas de maior concentração de raios, associadas com os núcleos da tempestade $\mathrm{T} 1$, e com a banda $\mathrm{B} 1$, foram deslocadas na direção NW-SW por cerca de $10 \mathrm{~km}$ das zonas de maior refletividade. Esses deslocamentos entre o CAD e o campo de refletividade nas imagens de radar foram observados em vários casos, quando a velocidade de translação passiva (VTP) do sistema de precipitação, determinada a partir dos dados de radar, alcançava valores elevados, tais como a VTP nesse dia, de $80 \mathrm{~km} / \mathrm{h}$.

A posição da fonte dos raios, i.é o sistema de precipitação, se transladado a $80 \mathrm{~km} / \mathrm{h}$, e a posição da descarga associada, acumulada no referencial fixo, i.é. CAD, feita no intervalo de \pm 15 minutos, centralizado no instante da imagem do radar, pode provocar uma diferença de $80 \mathrm{~km} / \mathrm{h} \times( \pm 0,25 \mathrm{~h}) \gg \pm 20$ $\mathrm{km}$ ao longo da direção da VPT (de $300^{\circ}$ a $110^{\circ}$ ). A velocidade de translação (Tabela 2) dos sistemas estudados varia de 32 à $125 \mathrm{~km} / \mathrm{h}$, com média de 70 $\mathrm{km} / \mathrm{h}$. Esta velocidade pode levar a uma diferença máxima de 17-18 km entre a posição da tempestade, no campo acumulado de descargas durante 0,5 hora, e a posição da tempestade na imagem do radar. Obviamente, o tempo de acumulação menor (e.g. 0,25 hora) diminui essa diferença. No entanto, observouse que a diminuição do tempo de integração prejudica a visualização da forma do sistema, em casos com baixa taxa de raios (e.g. em casos de convecção moderada e sistemas estratiformes).

A quantidade de raios registrados nos sistemas oscila periodicamente, alternando os períodos de baixas taxas de descargas com os períodos de altas taxas. Como se pode ver na figura $5 \mathrm{c}$, as imagens de radar dos sistemas não linear N1 (Fig. 5a) e linear (Fig. 5b) e o acúmulo de descargas foram feitos durante os mínimos relativos de taxa de descargas no

\begin{tabular}{|c|c|c|c|c|c|c|c|c|c|c|}
\hline \multirow[t]{2}{*}{$\mathrm{N}^{\circ}$} & \multirow[t]{2}{*}{$\begin{array}{l}\text { Data, } \\
1999\end{array}$} & \multirow{2}{*}{$\begin{array}{l}\text { Período de } \\
\text { observação } \\
\text { com radar, } \\
\text { hora local }\end{array}$} & \multirow[t]{2}{*}{$\begin{array}{l}\text { Padrão de } \\
\text { radar em } \\
240 \mathrm{~km}\end{array}$} & \multicolumn{3}{|c|}{$\begin{array}{l}\text { Raios no perído de } \\
\text { observação do sistema, área de } \\
\text { raio de } 480 \mathrm{~km}\end{array}$} & \multicolumn{2}{|c|}{$\begin{array}{l}\text { Taxa media de } \\
\text { raios, } \mathrm{h}^{-1}\end{array}$} & \multirow{2}{*}{$\begin{array}{l}\text { Trans } \\
\text { lação } \\
\text { VPT, } \\
\text { kmuh }\end{array}$} & \multirow{2}{*}{$\begin{array}{l}\text { Hora do } \\
\text { máximo } \\
\text { com } \\
\text { corrente } \\
\text { registrada } \\
\text { Trmax, }\end{array}$} \\
\hline & & & & sem & $+\mathrm{CG}$ & $-\mathrm{CG}$ & erm 480 & em 240 & & \\
\hline 1 & 2303 & $1943-2003$ & 0 & 0 & 0 & 0 & 0,0 & 0,0 & ND & 1948 \\
\hline 3 & 2805 & $1130-1853$ & SN, L2 & 2879 & 10 & 186 & 416,5 & 192,0 & 70 & 1853 \\
\hline 4 & 0906 & $0943-1622$ & SN & 72 & 113 & 17 & 30,4 & 2,0 & 45 & 1622 \\
\hline 5 & 2906 & $0941-1106$ & 0 & 0 & 0 & 0 & 0,0 & 0,0 & ND & ND \\
\hline 6 & 1407 & $1513-1749$ & SL & 72 & 14 & 7 & 35,7 & 0,0 & 80 & 1712 \\
\hline 7 & 1607 & $0941-1622$ & SN, SL & 69 & 62 & 37 & 25,1 & 10,6 & 85 & 1620 \\
\hline 8 & 1907 & $0952-1900$ & SL, SN & 5 & 17 & 8 & 3,3 & 0,3 & 32 & 1602 \\
\hline 9 & 2007 & $0856-1805$ & L2, SL & 271 & 1 & 12 & 28,4 & 15,7 & 40 & 1259 \\
\hline 10 & 2607 & 0800-1901 & Nl & 1768 & 295 & 2092 & 377,1 & 126,5 & 60 & 1044 \\
\hline 13 & 2907 & $0859-1903$ & N2, L2 & 4366 & 2 & 20 & 437,3 & 203,8 & 70 & 1308 \\
\hline 14 & 3007 & $0915-1140$ & L2, SL & 70 & 145 & 44 & 107,1 & 24,8 & 113 & 1047 \\
\hline 15 & 0608 & $1439-1807$ & $\mathrm{~N} 2, \mathrm{~L} 2$ & 262 & 3 & 2 & 77,0 & 8,65 & 62 & 1457 \\
\hline 16 & 1208 & $0906-1609$ & L2 & 74 & 1 & 17 & 13,0 & 10,9 & 72 & 1545 \\
\hline 17 & 2508 & $0956-1403$ & L2 & 29 & 2 & 1 & 7,8 & 0,0 & 85 & 1157 \\
\hline 18 & 2708 & $1153-1606$ & $\mathrm{~N} 2$ & 31 & 257 & 190 & 113,3 & 6,6 & ND & 1600 \\
\hline 19 & 3008 & $1224-1827$ & Ll(480) & 976 & 0 & 0 & 161,3 & 0,0 & 53 & 1827 \\
\hline 20 & 3108 & $0737-1651$ & Nl, Ll & 2071 & 252 & 56 & 257,6 & 188,7 & 90 & 1214 \\
\hline 21 & 0109 & $1000-1526$ & Nl, Ll & 2041 & 1227 & 77 & 615,6 & 352,6 & 75 & 1358 \\
\hline 22 & 0209 & $0936-1502$ & SL & 926 & 1110 & 74 & 387,1 & 10,45 & 65 & 1444 \\
\hline 23 & 0309 & $0827-1244$ & SN, L2 & 587 & 160 & 19 & 178,8 & 24,5 & 70 & 0859 \\
\hline 24 & 0709 & $0806-1940$ & Ll, Nl & 15103 & 1185 & 32 & 1410,9 & 743,1 & 125 & 1626 \\
\hline 25 & 0809 & 09241652 & SN & 182 & 483 & 91 & 101,2 & 0,3 & 83 & 0926 \\
\hline 26 & 1309 & $1433-1918$ & SL & 147 & 112 & 25 & 56,8 & 8,4 & 50 & 1917 \\
\hline \multirow[t]{3}{*}{27} & 1409 & $0948-1624$ & SN, L2 & 178 & 143 & 79 & 60,6 & 30,0 & 43 & 1201 \\
\hline & & soma & & 32179 & 5594 & 3086 & & & & \\
\hline & & média & & & & & 196,1 & 78,4 & 70,1 & \\
\hline
\end{tabular}

Tabela 2 - Padrões de radar e atividade elétrica dos sistemas de mesoescala de precipitação.

Table 2 - Radar patterns and electrical activity of mesoscale precipitation systems. 
circulo de $480 \mathrm{~km}$, quando a quantidade de descargas registradas em 5 min variou em torno de 8-10, e 2025 , respectivamente. Estas taxas mínimas $(\mathrm{min})$ se alternam nos instantes onde a produção de raios aumenta em 2-2,5 vezes, alcançando os máximos (max) de 40-45 descargas em 5 minutos. Estes máximos aparecem em períodos de cerca de $70 \mathrm{~min}$ e, obviamente, são associados ao ciclo natural de convecção auto-organizada na escala b (e.g. Abdoulaev, 1995, entre outros).

Considerando-se que pode haver uma desfasagem entre o instante da observação da refletividade máxima, Zmax, do sistema e o instante em que ocorre a taxa máxima de raios, foi procurado um instante padrão no qual todos os sistemas poderiam ser comparados univocamente. Observouse que durante o período de observação do sistema, a extensão vertical de $Z=30 \mathrm{dBZ}$ (essa refletividade é observada na maioria dos sistemas), a altura H30 teve uma variação significativa, alcançando seu máximo em intervalo próximo ao instante de refletividade máxima $\mathrm{Zmax}$. $\mathrm{O}$ instante do valor máximo de $\mathrm{H} 30$ foi considerado como instante da intensidade máxima do sistema, Tmax (Tabela 2). Dessa forma, os parâmetros do radar e as taxas de descargas elétricas foram calculados para um período de \pm 15 minutos próximos a este instante.

$\mathrm{Na}$ tabela 3 nota-se que as alturas de $\mathrm{H} 30$ variam de um sistema para outro, alcançando os níveis de 11 à 16 km (coluna 4), nos sistemas severos. Nesses casos, a altura $\mathrm{H} 30$ foi $7 \mathrm{~km}$ maior do que a altura correspondente a $0^{0} \mathrm{C}$ (coluna 5). Em geral, nos sistemas com alturas maiores do que $10 \mathrm{~km} \mathrm{a}$ refletividade é maior do que $45 \mathrm{dBZ}$ (coluna 3 ). A área total ocupada pela precipitação (ST, coluna 6) varia muito de um sistema para outro, registrando-se $9.000 \mathrm{~km}^{2}$ no sistema L2 no dia 25/08 e mais de $142.000 \mathrm{~km}^{2}$ no sistema L2-SL no dia $28 / 05$. Um sistema "médio" no instante de máxima atividade apresentou um campo de precipitação de $35.000 \mathrm{~km}^{2}$ (coluna 6) com núcleo de refletividade $>30 \mathrm{dBZ}$ de $6.000 \mathrm{~km}^{2}$ (coluna 7), onde as alturas H30 de 8,8 $\mathrm{km}$ penetram quase $5 \mathrm{~km}$ na camada atmosférica com temperaturas negativas, $\mathrm{DH}$ (coluna 5). A taxa de raios produzida pelos sistemas (colunas 8-11) num círculo de raio de $480 \mathrm{~km}$, variam de 0 , no sistema L2, no

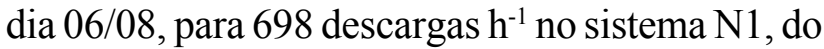

dia 26/07. Notou-se que a taxa de raios no instante Tmax pode ser bastante diferenciada dos valores médios na tabela 2. Embora os sistemas produzam, em média, no instante Tmax, uma quantidade de descargas 1,5 vezes maior do que as taxas médias durante toda a observação, em certos casos observam-se uma diminuição da taxa de até $40 \%$, como no caso do sistema severo no dia 07/09, e um aumento de até 6 vezes, no caso do sistema estratiforme no dia 08/09. Um sistema "médio" produz $84,8(220,6)$ descargas no círculo de raio $240(480) \mathrm{km}$, o que corresponde a uma densidade equivalente de 4,7 e $3 \times 10^{-4}$ descargas $\mathrm{x}\left(\mathrm{h} \mathrm{km}^{2}\right)^{-1}$. Observando-se a pequena variação (menor que $<1,8$ ) da quantidade de descargas no círculo de 240 a 320 km (coluna 11), ou entre os círculos de 320 e 480 $\mathrm{km}$ pode-se resumir que em 20 de 24 dos casos, todo o sistema com raios foi observado no círculo de $320 \mathrm{~km}$ do radar, provando as suposições anteriores sobre o tamanho limite do sistema.

A análise visual dos dados da Tabela 3 demostra que a taxa de raios cresce com a altura de $\mathrm{H} 30$ e com a refletividade. $\mathrm{Na}$ tabela 4 são mostradas as taxas de descargas em relação a altura (colunas 2-4) e a refletividade (colunas 5-7). Verificou-se que com o aumento inicial da extensão DH de 2 a $3 \mathrm{~km}$, as taxas de descargas cresceram bruscamente. Enquanto os sistemas com $\mathrm{H} 30$, entre 5 e $6,5 \mathrm{~km}$, produziram 8,5 descarga $^{-1}$ no círculo de $240 \mathrm{~km}$, em média, os sistemas com alturas entre 6,5 e 7,5 km produziram 10 vezes mais. Estes resultados, permitem preestabelecer que a altura de $30 \mathrm{dBZ}$, obtida de dados do radar, é um bom indicador da atividade de descargas à terra na região de estudo. McGorman \& Filiaggi (1997) notaram que a taxa das descargas com correntes grandes, cresce gradualmente com extensão de H30 para temperaturas negativas (a aplicação de outras variáveis, e.g. refletividade máxima ou quantidade d'água integrada, leva à subestimação das descargas de grandes correntes). Segundo os autores citados, somente $8 \%$ das células com $\mathrm{DH}>9 \mathrm{~km}$ não produziram descargas à terra. Na tabela 4 , as nuvens cuja camada negativa foi de aproximadamente $9 \mathrm{~km}$, apresentaram uma taxa em 2 vezes maior do que às nuvens com camada de $3 \mathrm{~km}$. Nos sistemas agrupados pela refletividade o aumento brusco da taxa 


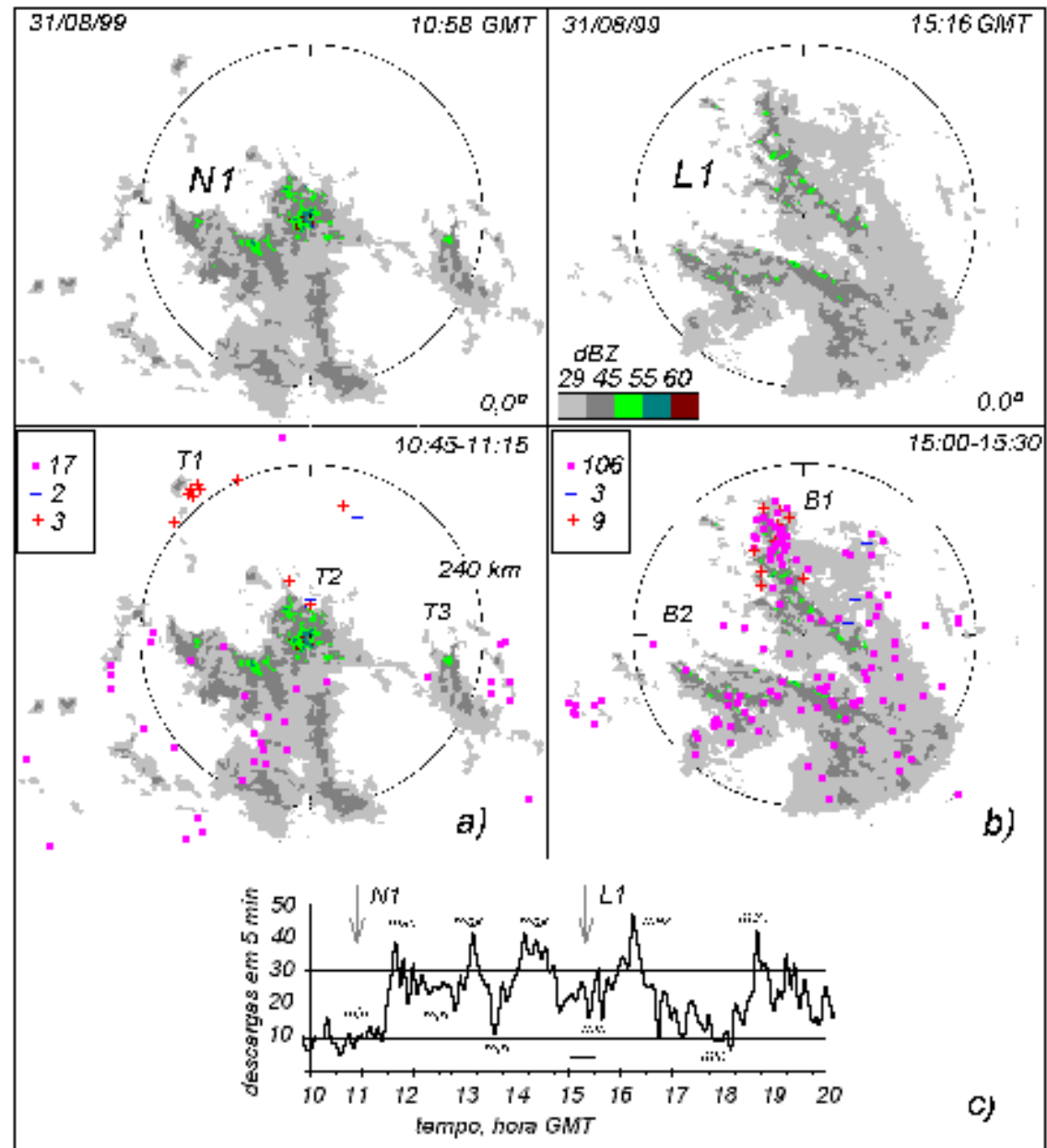

Figura 5 - Atividade elétrica dos sistemas convectivos severos não linear N1 (a) e linear L1 (b) no dia 31/08/99. No instante (a) observouse o máximo local das alturas do eco de radar e queda de granizo de $1 \mathrm{~cm}$ de tamanho na tempestade T2 de baixa produção dos raios; descargas positivas e negativas são marcadas convencionalmente; quadrados são descargas de polaridade não determinada; c) oscilações periódicas de intensidade de descargas no raio de abrangência de radar do $480 \mathrm{~km}$.

Figure 5 - Electrical activity of the severe nonlinear N1 (a) and linear L1 (b) convective system on 31/08/99. At time of image (a), the local maximum of radar echo height and hail of $1 \mathrm{~cm}$ was observed on the poor lightning storm T2; positive and negative flashes are demarcated by conventional symbols; squares are non determined polarity flashes; c) periodical oscillation of 5-min cloud-to-ground flash intensity in $480 \mathrm{~km}$ range of radar.

de 38 a 310 descargas por hora ocorre quando a refletividade supera $50 \mathrm{dBZ}$.

No estágio Tmax do sistema o aumento da altura e da refletividade geralmente é acompanhado pelo aumento da área total do eco ST e a área com refletividade maior do que $30 \mathrm{dBZ}$ (veja S30 na Tabela 4). Comparando-se a taxa média em círculos de 240 a $320 \mathrm{~km}$ do radar (onde foi observado mais de um sistema) pode-se estimar a eficiência de produção de raios relativa à unidade de área. A área unitária do sistema com alturas $>7,5 \mathrm{~km}$ produz oito vezes mais $\left(0,00495\right.$ descarga $\left.\left(\mathrm{h} \mathrm{km}^{2}\right)^{-1}\right)$ descargas do que aquela do sistema de $\mathrm{H} 30 £ 6,5 \mathrm{~km}$. A taxa de 0,0052 descarga $\left(\mathrm{h} \mathrm{km}^{2}\right)^{-1}$ produzida pela área com $Z>50$ $\mathrm{dBZ}$ é seis vezes maior do que a taxa de $1 \mathrm{~km}^{2} \mathrm{com}$ $\mathrm{Z}<50 \mathrm{dBZ}$. A produção de descargas relativa a $\mathrm{S} 30$ dos sistemas com Z $>50 \mathrm{dBZ}$ e $\mathrm{H} 30>7,5 \mathrm{~km}$ é 2,5 vezes maior $\left(0,0245\right.$ descarga' $\left.\left(\mathrm{h} \mathrm{km}^{2}\right)^{-1}\right)$ do que a dos sistemas de alturas $£ 7,5 \mathrm{~km}$ e refletividade $<50$ $\mathrm{dBZ}$, cuja produção tem pequena variação, entre 0,0072 à 0,0113 descarga' $\left(\mathrm{h} \mathrm{km}^{2}\right)^{-1}(0,0093 \mathrm{em}$ media).

O crescimento observado da produção com a altura e a refletividade explica as altas taxas dos sistemas severos L1 eN1. A quantidade significativa de raios detectáveis nos sistemas compostos das linhas de convecção moderada e vasta região estratiforme (e, consequentemente maior número total de descargas de grandes correntes, vide Rutledge e McGorman, 1988), explica-se em razão das alturas de H30 terem sido $1 \mathrm{~km}$ maior do que em sistemas estratiformes ou bandas convectivas, separadamente. 


\begin{tabular}{|c|c|c|c|c|c|c|c|c|c|c|}
\hline \multirow[t]{2}{*}{$\begin{array}{l}\text { Data, } \\
1999\end{array}$} & \multirow[t]{2}{*}{$\begin{array}{l}\text { Padrão } \\
\text { de eco }\end{array}$} & \multirow[t]{2}{*}{$\begin{array}{l}Z \\
\text { máxima } \\
\mathrm{dBZ}\end{array}$} & \multirow{2}{*}{$\begin{array}{c}\text { Altura } \\
\mathrm{Z}=30 \\
\mathrm{dBZ}, \mathrm{H} 30 \text {, } \\
\mathrm{km}\end{array}$} & \multirow[t]{2}{*}{$\begin{array}{l}\text { Camada H30 } \\
<0^{\circ} \mathrm{C} \\
\mathrm{\Delta H}, \mathrm{km}\end{array}$} & \multicolumn{2}{|c|}{$\begin{array}{c}\text { área total de eco, ST, } \\
\text { e com } Z=30 \mathrm{~dB} Z \text {, } \\
\mathrm{S} 30, \mathrm{~km}^{2}\end{array}$} & \multicolumn{4}{|c|}{$\begin{array}{l}\text { Taxa de raios no intervalo } \pm 15 \\
\text { minutos de } T \max h^{-1}\end{array}$} \\
\hline & & & & & ST & S30 & $\begin{array}{r}\mathrm{em} \\
480 \\
\end{array}$ & $k \%$ & $\begin{array}{l}\mathrm{em} \\
240\end{array}$ & $\begin{array}{l}\text { em } \\
320\end{array}$ \\
\hline 2303 & 0 & 50 & 11,5 & 6,4 & 24942 & 349 & 0 & na & 0 & 0 \\
\hline 2805 & SN, L2 & $=50$ & 7,2 & 3,0 & 141594 & 31501 & 632 & 147 & 348 & 374 \\
\hline 0906 & SN & $=35$ & 7,5 & 4,5 & 78233 & 9790 & 58 & 190 & 18 & 56 \\
\hline 2906 & 0 & $<30$ & na & na & 2694 & 0 & 0 & na & 0 & 0 \\
\hline 1407 & SL & $=30$ & 5,0 & 1,1 & 10872 & 183 & 14 & 39 & 0 & 0 \\
\hline 1607 & SN, SL & $=40$ & 6,0 & 2,6 & 43858 & 4478 & 16 & 64 & 2 & 2 \\
\hline 1907 & SL, SN & $=35$ & 5,5 & 2,2 & 17437 & 1549 & 18 & 90 & 0 & 0 \\
\hline 2007 & L2, SL & $=45$ & 6,2 & 2,9 & 15815 & 1052 & 26 & 90 & 22 & 22 \\
\hline 2607 & N1 & $=55$ & 12,5 & 8,8 & 61526 & 11588 & 698 & 143 & 316 & 414 \\
\hline 2907 & $\mathrm{~N} 2, \mathrm{~L} 2$ & $=45$ & 13,0 & 9,3 & 28217 & 5658 & 580 & 130 & 44 & 196 \\
\hline 3007 & L2, SL & $=45$ & 12,0 & 8,1 & 34131 & 12896 & 140 & 131 & 2 & 20 \\
\hline 0608 & $\mathrm{~N} 2, \mathrm{~L} 2$ & $=30$ & 6,0 & 1,8 & 9662 & 487 & 48 & 62 & 12 & 18 \\
\hline 1208 & L2 & $=45$ & 6,5 & 2,9 & 13210 & 1222 & 44 & 338 & 34 & 42 \\
\hline 2508 & L2 & $=30$ & 5,5 & 1,5 & 8870 & 889 & 14 & 179 & 0 & 0 \\
\hline 2708 & N2 & $=45$ & 10,6 & 7,4 & 26843 & 1950 & 102 & 90 & 30 & 78 \\
\hline 3008 & $\mathrm{L1}(480)$ & $=50$ & 10,0 & 5,7 & 12980 & 1886 & 184 & 114 & 0 & 0 \\
\hline 3108 & N1, L1 & $=60$ & 11,5 & 7,2 & 104037 & 26048 & 264 & 102 & 230 & 248 \\
\hline 0109 & N1, L1 & $>55$ & 15,5 & 11,9 & 46437 & 5376 & 594 & 96 & 332 & 494 \\
\hline 0209 & SL & $=35$ & 6,8 & 2,5 & 19350 & 2913 & 244 & 58 & 18 & 158 \\
\hline 0309 & SN, L2 & $=45$ & 7,0 & 3,5 & 64991 & 14050 & 236 & 132 & 75 & 94 \\
\hline 0709 & $\mathrm{~L} 1, \mathrm{~N} 1$ & $>60$ & 16,0 & 11,4 & 47749 & 11936 & 536 & 36 & 502 & 506 \\
\hline 0809 & SN & $=35$ & 6,6 & 3,3 & 21505 & 1860 & 570 & 580 & 0 & 30 \\
\hline 1309 & SL & $=35$ & 7,0 & 2,7 & 17437 & 1549 & 260 & 119 & 42 & 52 \\
\hline 1409 & SN, L2 & $>45$ & 7,0 & 2,9 & 20016 & 2931 & 236 & 386 & 94 & 94 \\
\hline & & & & & & & & & & \\
\hline & media & & 8,8 & 4,9 & 34896 & 6086 & 220,6 & 150 & 84,8 & 115,9 \\
\hline
\end{tabular}

Tabela 3 - Sistema de mesoescala durante estágio de parâmetros máximos de radar.

Table 3 - Mesoscale systems during stage of maximum in radar parameters.

Do ponto de vista da organização dos sistemas, Robinson e Biggerstaff (1997) observaram que os sistemas com convecção na forma linear acompanhados por região estratiforme tendem a produzir maior quantidade de descargas à terra do que os sistemas não organizados linearmente. Isso particularmente é obvio na análise do gráfico da Fig. $5 \mathrm{c}$, que mostra o aumento geral da taxa de raio na linha de convecção severa. Os sistemas estratiformes com banda convectivas moderadas podem ser um estágio de dissipação da linha de convecção severa, sistemas de dinâmica estreitamente organizada, que efetivamente separa as cargas positivas e negativas por longo tempo (vide Houze, 1993).

\section{CONCLUSÕES}

A atividade elétrica dos sistemas sinóticos e de mesoescala observados por radar meteorológico em Pelotas, RS, e pelo satélite GOES-8, em 27 dias do outono e inverno de 1999, numa região ciclogenética do Sudeste da América do Sul, foi examinada baseando-se nos dados sobre a localização e o instante de descarga de retorno arquivados na rede integrada de descargas atmosféricas à terra (RIDAT). Os Campos Acumulados das Descargas (CAD) à terra de 0,5 a 1 hora, grade $15^{\prime} 15 \mathrm{~km}$, apresentaram informações sobre a orientação e o movimento das frentes (16 casos) de qualidade comparável ou até mais detalhada do que aquela mostrada pelas imagens de satélite (Fig. 1). A sobreposição dos campos acumulados de 2 horas, com código de tempo em cores (Fig. 2), permitiram acompanhar as frentes situadas sobre o Uruguai e sobre o oceano Attântico, até 400 quilômetros longe da região de maior eficiência na detecção de raios da RIDAT. O número de descargas registradas nos 9 casos de ciclogênese local foi de 1600 descargas $h^{-1}$, o que representou 4 vezes mais do que o número de descargas associado aos sistemas transientes. Baseando-se na análise de seqüência contínua dos CAD de 30 minutos, nos casos de ciclogênese e de frentes lentas, (Fig. 2 e 


\begin{tabular}{|c|c|c|c|c|c|c|}
\hline \multirow[t]{2}{*}{ Parâmetros médios } & \multicolumn{3}{|c|}{ Alturas de $\mathrm{Z}=30 \mathrm{dBZ}, \mathrm{H} 30, \mathrm{~km}$} & \multicolumn{3}{|c|}{ Refletividade máxima, dBZ } \\
\hline & $\begin{array}{c}\mathrm{H} 30 \leq 6 \\
5\end{array}$ & $6,5<\mathrm{H} 30 \leq 7,5$ & $\mathrm{H} 30>7,5$ & $Z \leq 40$ & $40<Z \leq 50$ & $Z>50$ \\
\hline$\Delta \mathrm{H}, \mathrm{km}$ & 2,1 & 3,2 & 8,7 & 2,5 & 4,9 & 7,8 \\
\hline $\begin{array}{l}\text { Taxa de descargas em raio de } \\
\qquad 480 \mathrm{~km}^{-1} \mathrm{~h}^{-1}\end{array}$ & 19,0 & 350,0 & 364,5 & 136,2 & 169,2 & 415,5 \\
\hline $\begin{array}{l}\text { Taxa de descargas em raio de } \\
\qquad 320 \mathrm{~km}, \mathrm{~h}^{-1}\end{array}$ & 12,0 & 122,6 & 244,5 & 34,9 & 68,5 & 339,3 \\
\hline $\begin{array}{l}\text { Taxa de descargas em raio } \\
\qquad 240 \mathrm{~km}^{-1} \mathrm{~h}^{-1}\end{array}$ & 8,5 & 85,0 & 203,0 & 10,0 & 33,6 & 310,5 \\
\hline Area total de eco, ST, $\mathrm{km}^{2}$ & 17100 & 51875 & 45240 & 26195 & 30885 & 62750 \\
\hline $\begin{array}{l}\text { Taxa media na ST, } \\
10^{-4} \text { descarga } \times \mathrm{h}^{-1} \times \mathrm{km}^{-2}\end{array}$ & 6,0 & 20,0 & 49,5 & 8,5 & 16,5 & 52,0 \\
\hline Area de $\mathrm{Z}>30 \mathrm{dBZ}, \mathrm{S} 30, \mathrm{~km}^{2}$ & 1410 & 9220 & 9670 & 2400 & 5530 & 12620 \\
\hline $\begin{array}{c}\text { Taxa media na } \mathrm{S} 30, \\
10^{-4} \text { descarga } \times \mathrm{h}^{-1} \times \mathrm{km}^{-2}\end{array}$ & 72,5 & 112,6 & 231,4 & 93,5 & 92,3 & 257,5 \\
\hline Número de casos & 7 & 7 & 8 & 9 & 8 & 7 \\
\hline
\end{tabular}

Tabela 4 - Densidade de raios à terra vs. altura e refletividade de precipitação.

Table 4 - Cloud-to-ground flash density vs. height and refletivity of precipitation system

Fig. 3), foi revelado que, em CAD, as seções frontais quente e fria adquirem a forma convexa e côncava respetivamente, com diferenças na polaridade preferencial das descargas produzidas, dando os maiores detalhes sobre o estágio do ciclone do que as imagens do GOES-8 (Fig. 4). Observou-se ainda que em certos estágios do desenvolvimento as aglomerações das nuvens altas não produzem descargas à terra detectáveis pelo sistema de observação.

A análise comparativa entre campos de refletividade e atividade elétrica dos 37 sistemas de escala $\mathrm{b}(<300 \mathrm{~km})$, classificados em seis padrões, demonstra a diferença significativa entre as taxas de descargas associadas com certo tipo de sistema de precipitação. Os sistemas convectivos severos (L1, N1), e as bandas de convecção moderada acompanhadas por região estratiforme (L2-SN, L2SL) têm taxas de descargas 5-6 vezes maior do que a média (Tabela 2).

Devido à variação significativa na produção de raios entre os estágios de um sistema, e os ciclos periódicos de 1 hora dentro de um estágio (Fig. 5), a atividade elétrica dos sistemas foi comparada no instante de altura máxima de eco com $Z=30 \mathrm{dBZ}$, $\mathrm{H} 30$, o que demonstrou ser um bom indicador da atividade elétrica do sistema de precipitação (Tabela 3). Por outro lado, as taxas de descargas na unidade de área de eco (Tabela 4) aumentaram 2-3 vezes com o aumento de $\mathrm{H} 30$ por $1 \mathrm{~km}$ da camada atmosférica, $\mathrm{DH}$, com temperaturas $<0^{\circ} \mathrm{C}$. Os sistemas com alturas $<7,5 \mathrm{~km}$, refletividade $<50 \mathrm{dBZ}$, e DH de 3 à $5 \mathrm{~km}$ produziram, durante uma hora, em torno de 90 descargas na área equivalente a $10^{4} \mathrm{~km}^{2}$ ocupada pela refletividade $Z>30$, enquanto os sistemas com $Z>50$ $\mathrm{dBZ}$ e alturas $>7,5 \mathrm{~km}$, na mesma área, produziram 230-260 descargas por hora.

Os resultados encontrados neste trabalho demonstram a viabilidade e as limitações do acompanhamento operacional dos sistemas de mesoescala na região sudeste da América do Sul e parte do Oceano Atlântico adjacente, empregandose técnicas apropriadas de integração de dados de descargas à terra, radar e satélite.

\section{AGRADECIMENTOS}

Os autores agradecem aos colegas da Assessoria de Planejamento de Informação de FURNAS pelo fornecimento dos dados de descargas à terra, ao Corpo Editorial da Revista Geofisica pelas sugestões construtivas, ao CNPq (processos 301738/95-5 e 381945/01-4) e à FAPERJ (processo 151135/ $00)$ pelo apoio.

\section{REFERÊNCIAS}

Abdoulaev, S., Marques, V. S., Pinheiro, F. M. A., Martinez, E. F. A. \& Lenskaia, O., 2000. An exploratory study of lightning activity on 
southeast and south of Brazil. 20th Conf. on Severe Local Storms. Amer. Met. Soc., 11-15, September, 2000, P.5.2, 110-111, Orlando, Florida:

Abdoulaev, S., 1995. Evolução e hierarquia das aglomerações de Cumulonimbus. Rev. Bras. Met., 10 (1):1-9.

Abdoulaev, S. \& Lenskaia, O., 1998. Evolução das Linhas de Convecção Severa. Parte 1. Classificação. Revista Brasileira de Meteorologia ,13(2):15-36.

Abdoulaev, S., Starostin, A., Lenskaia, O. \& Gomes, R.G., 1998. Sistemas de Mesoescala de Precipitações no Rio Grande do Sul. Parte 1: Classificação dos Sistemas de Mesoescala de Precipitações. Revista Brasileira de Meteorologia, 13(2):57-74

Beneti, C. A.A., Leite, A.A., Garcia, E. A., Assunção, S. A. M., Cazetta Filho, L. A R. \& Januario dos Reis, R., 2000. RIDAT - Rede Integrada de detecção de Descargas Atmosféricas no Brasil: Situação atual, aplicações e perspectivas. XI Congr. Bras. Met., 19711979, Rio de Janeiro, RJ

Browning, K. A., 1989. The mesoscale database and its use in mesoscale forecasting. Quartly Journal Royal Met. Soc., 115: 717-762.

Christian, H.J \& Latham, J., 1998. Satellite measurements of global lightning. Quartly Journal Royal Met. Soc., 124: 1771-1773.

Cotton, W. R., \& Anthes, R. A., 1989. Storm and Cloud Dynamics. International Geophysics Series, v. 44, Academic Press, London, 883 pp.

Furnas, 1999. Sistema de detecção de descargas atmosféricas de FURNAS. Relatório Interno de FURNAS Centrais Elétricas, 1999, disponível na Assessoria de Planejamento, Rio de Janeiro, 8 pp.

Gin R. B. B. \& Guedes, R. L., 2000. Climatologia de relâmpagos no Brasil: Análise preliminar. XI Congr. Bras. Met., 1948-1957, Rio de Janeiro, RJ

Gin, R.B.B, Pereira Filho, A. J. \& Silva Dias, M. A. F., 1998. Estudo das descargas elétricas atmosféricas em sistemas convectivos organizados: Análise preliminar. X Congr. Met., Brasilia, 1998, MR98009, CD-ROM.
Gin, R.B.B, Pereira Filho, A. J., Beneti, C.A.A. \& Guedes R. L., 2000. Estudo das descargas elétricas atmosféricas no Sul e Sudeste do Brasil: Análise preliminar. XI Congr. Bras. Met., 20122020, Rio de Janeiro, RJ

Gin, R.B.B., Pinto I.R.C.A., Pinto Jr., O. \& Mendes Jr., O., 1996. Estudo de relâmpagos nuvem solo no Estado de Minas Gerais associados às condições meteorológicas. IX Congr. Bras. de Met., 1,17-18, Campos do Jordão, SP.

Hodanish, S., Sharp, D., Collins, W., Paxton, C., Orville, R. E., 1997. A 10-yr monthly lightning climatology of Florida: 1986-95. Weather and Forecasting, 12: 439-448.

Houze, R.A., 1993. Cloud dynamics. Academic Press., London, 573 pp.

Ludlam, F.H. 1980. Cloud and Storms. The behavior and effect of water in the atmosphere. Pennsylvania State Univ., 405 pp.

Macgorman, D. R. \& Fillagi, T. 1997. Lightning ground flash rates relative to radar-inferred storm properties. 28th Conf. on radar meteorology. AMS, September 7-12, 1997,143-144, Austin, Texas.

Macgorman, D. R., K. C. Crawford \& Xia, H., 1993. A lightning strike climatology for Oklahoma. Conference on Atmospheric Electricity. AMS, October 4-8, 1993, 768-773, St.Luis, Missouri.

Mason, B. J., 1971. The Physics of Clouds. Oxford University Press.,671 pp.

Matveev, L. T., 1984. The Course of General Meteorology (Physics of Atmosphere). Hydrometizdat, Leningrado. Chapter 23. Atmospheric Electricity, 752 pp.

Mazin, I. P \& Khrgian, A. K.H., 1989. Oblaka e oblachnaia atmosfera. (Handbook of Clouds and Cloudy Atmosphere.) Hydrometizdat, Leningrado. 646 pp.

Orville, R. E. \& Silver, A. C., 1997. Lightning ground flash density in contiguos United States:1992-1995. Monthly Weather Review, 125: 631-638.

Pinto JR., O., Gin, R.B.B, Pinto, I.R.C.A, Mendes, O. JR., Diniz J. H \& Carvalho A. M., 1996. Cloud-to-ground lightning flash characterististics in southeeastern Brasil for 1992- 
1993 summer season. J. Geophys. Res., 101(D23): 29627-29635.

Reap, R. M. 1993. Lightning distributions associated with synoptic map types. Conference on Atmospheric Electricity. AMS, October 48,1993, 761-767, St. Luis, Missouri.

Robinson, M., \& Biggerstaff, M. I., 1997. Relationship between reflectivity profiles, cloudto-ground lightning, and storm system characteristics for convective cells in a coastal zone. 28th Conference on Radar Meteorology, AMS, September 7-12, 1997, 153-154, Austin, Texas.

Roohr, P. B. \& Vonder Haar, T.H., 1994. A comparative analysis of temporal variability of lightning observation and GOES imagery. Journal of Applied Meteorology, 33:1271-1290.
Rutledge, S. A. \& Macgorman, D. R., 1988. Cloud-to-ground Lightning Activity in the 10-11 June 1985 Mesoscale Convective System observed during the Oklahoma -Kansas PRESTORM project. Monthly Weather Review, 116:1393-1408.

Silva Dias, M. A. F., 2001. Experimento de grande escla da interação biosfera- atmosfera na Amazônia: resultados preliminares. Boletim de Sociedade. Brasileira de Meteorologia, 25 (1):714.

Zavialov, P. O., Wainer, I., \& Absy, J.M., 1999. Sea surface temperature variability off Southern Brazil and Uruguay as revealed from historicla data since 1854. Jornal of Geophisical Res., 104, 2102121032.

\section{ANALYSIS OF MESOSCALE SYSTEM USING CLOUD-TO-GROUND FLASHDATA.}

The aim of the present work is to analyze the possibility of using the lightning data for monitoring and diagnosis mesoscale and large scale precipitation systems in Southern Atlantic ciclogenetic region associating with cold/warm oceanic currents confluence, between $35^{\circ} \mathrm{S}$ to $30^{\circ} \mathrm{S}$, some hundred km southward of high density Brazilian lightning detection network (Fig. 1a).

Meteorological radar, geostationary satellite and cloud-to-ground-flash data are used to analyze different types of cold season extratropical thunderstorms. During the period of 27 days (543 hours), May to September 1999, the cloud-to-ground location network, mainly composed by time-of arrival detectors, recorded 446000 cloudto-ground flashes (polarity of $50 \%$ of the flashes was also determined). Day-to-day registered flashes numbers vary from less than one thousand to one hundred thousand (Table 1). Generally the thunderstorms are more intense when synoptic situation was determined by local cyclogenesis in Southern Brazil ( 9 cases). Thunderstorms in these situations produced about 1600 cloud-to-ground flashes in one hour. Despite of that, in frontal situation (16 cases), hourly recorded flash rate is four times less than cyclones.

Orientation and velocity of fast-moving fronts could be determined from one hour Time-Integrated Lightning Field (TILF), grid 15 x $15 \mathrm{~km}$, with same quality as geostationary satellite images (Fig. 1b). The straight bands of frontal flashes (Fig. 1c) can be detected in a vast continental and oceanic area around southern Brazil and can be followed operationally by using time color-coded display with 2 hours TILF superposition (Fig. 2).

Frequently, in the presence of local cyclogenesis, the superposition of 1-2 hours TILF formed very complex images (Fig. 2 and Fig. 3). If it is a case, the system evolution can be analyzed using the sequence of 30 minutes time integrated lightning fields, timely centralized with infrared satellite images (Fig 4). The warm (Q) and cold front (F) sections of cyclone can be recognized from this TILF sequence as concave and convex arcs, respectively. The preferential negative polarity of flashes in convective and positive polarity in stratiform regions can be used to more detail about of frontal type (Fig. 4).

The organization of mesoscale precipitation systems on Southern Brazil was object of preceding studies. The 37 mesoscale systems observed at Pelotas University radar $\left(31^{\circ} 43^{\prime} \mathrm{W}, 52^{\circ} 18^{\prime} \mathrm{W}\right)$ were classified according to the degree of spatial organization and intensity of radar echoes as: 4 severe squall lines L1, 4 severe nonlinear, N1, 10 moderate convective bands L2, 3 moderate sparse thunderstorms N2, 7 linear and 7 nonlinear stratiform precipitation, SL and SN respectively (Table 2) 
The flash density depend on, critically, both the intensity and organization of system (Fig. 5). It was observed that severe convective systems $(\mathrm{L} 1, \mathrm{~N} 1)$ have biggest values of hourly mean flash quantity in 480 range of radar, five-six times more than the mean value of all systems. During system evolution the flash intensity oscillates, alternating periods of relatively weak and strong flash rates (Fig. 5). The moderate convective bands, if then are accompanied by stratiform regions (L2-SN, L2-SL), can achieve pronounced flash rates, also.

The time variations of flash intensity lead to some subjectivity in estimation of short-time cloud-toground flash productivity of mesoscale system. For objectivity, the system flash rates were compared for period of 30 minutes around the time, $\mathrm{T}$ max, when it was observed radar reflectivity maximum, $\mathrm{Z}$ max, and maximum height of $30 \mathrm{dBZ}, \mathrm{H} 30$ (Table 3). These H30, that vary up to $6.5 \mathrm{~km}$ in stratiform systems, and up to $15 \mathrm{~km}$, during severe convection, can be considered as good indicator of flash intensity. The equivalent hourly flash density for square kilometer of radar echo increases 2 to 3 times when $\mathrm{H} 30$ increases only $1 \mathrm{~km}$ toward negative temperatures (Table 4). The areas of $10000 \mathrm{~km}^{2}$ occupied by echo with Z $>30 \mathrm{dBZ}$ associated with mesoscale systems, height $\mathrm{H} 30$ (less our equal to $7.5 \mathrm{~km}$ ) and reflectivity less than $50 \mathrm{dBZ}$, produced about 90 hourly recorded cloud-toground flashes, while the same area of systems with reflectivity more than $50 \mathrm{dBZ}$ and heights $>7.5 \mathrm{~km}$, produced between 230 and 260 flashes. Generally, the observed height/flash density dependence, can explain why two type of systems, compact severe and the moderate convection combined with vast trailing stratiform region, have major number of recorded flashes than others systems.

\section{NOTE ABOUT THE AUTHORS}

\section{Sanjar Abdoulaev}

M. S. (physics), physics of sea and land water, from Moscow State University, Moscow, 1984. Ph.D. (physics and mathematics), meteorology, at Hydrometeorological Research Center of Russia, Moscow, 1992. Engineer-meteorologist at Weather Modification Service of Moldavia, Moldavia, 1984-1993. Visiting researcher and professor at Universidade Federal de Pelotas, Pelotas, RS, Brazil, 1993-2000. Visiting researcher at Universidade Estadual Norte Fluminense, Rio de Janeiro, RJ, Brazil, 2000-2002. Areas of interest: Mesoscale and Radar Meteorology, Severe Local Storms.

\section{Valdo da Silva Marques}

Graduação em Meteorologia e Física, em 1968 e 1969, respectivamente, pela Universidade Federal do Rio de Janeiro, Rio de Janeiro, RJ. Mestrado em Hidráulica e Saneamento pela Universidade de São Paulo, São Carlos, SP, em 1973. Doutorado em Meteorologia pelo Instituto de Pesquisas Espaciais, São José dos Campos, SP, em 1981. Pós - doutorado pela Universite Blaise Pascal, Clermont Ferrand, França, período de 1985 a 1986. Atualmente Professor da Universidade Estadual Norte Fluminense, em Macaé, RJ.

\section{Francisca Maria Alves Pinheiro}

Graduada em Meteorologia, em 1968, pela Universidade Federal do Rio de Janeiro, Rio de Janeiro, RJ. Mestrado em Meteorologia Sinótica pela Florida State University, Talahassee, Estados Unidos,em 1975. Doutorado em Agrometeorologia pela Macquarie University, Sydney, Australia, em 1989. Atualmente Pesquisador Visitante, da Universidade Estadual Norte Fluminense, Macaé, RJ, como bolsista da FAPERJ .

\section{Eduardo Fernandes Alonso Martinez}

Formado em Engenharia Eletrônica, Universidade Gama Filho, Rio de Janeiro, em 1992. Especialização em Climatologia pela Universidade do Estado do Rio de Janeiro, Rio de Janeiro, RJ, em 1994. Atualmente Bolsista do CNPq, no Sistema de Meteorologia do Estado do Rio de Janeiro, RJ.

\section{Olga Lenskaia}

M. S. (physics), atmospheric physics, from the Moscow State University, Moscow, 1984. M. S.(meteorology) from Universidade Federal de Pelotas, Pelotas, RS, 1998. Engineer-meteorologist at Weather Modification Service of Moldavia, Moldavia, 1984-1993. Professor at Universidade Federal de Pelotas, Pelotas, RS, Brazil, 1997-2000. Areas of interest: Mesoscale and Radar Meteorology, Severe Local Storms. 